\title{
BCFT entanglement entropy at large central charge and the black hole interior
}

\author{
James Sully, Mark Van Raamsdonk and David Wakeham \\ Department of Physics and Astronomy, University of British Columbia, \\ Vancouver, BC V6T 0C2, Canada \\ E-mail: sully@phas.ubc.ca, mav@phas.ubc.ca, daw@phas.ubc.ca
}

ABSTRACT: In this note, we consider entanglement and Renyi entropies for spatial subsystems of a boundary conformal field theory (BCFT) or of a CFT in a state constructed using a Euclidean BCFT path integral. Holographic calculations suggest that these entropies undergo phase transitions as a function of time or parameters describing the subsystem; these arise from a change in topology of the RT surface. In recent applications to black hole physics, such transitions have been seen to govern whether or not the bulk entanglement wedge of a (B)CFT region includes a portion of the black hole interior and have played a crucial role in understanding the semiclassical origin of the Page curve for evaporating black holes.

In this paper, we reproduce these holographic results via direct (B)CFT calculations. Using the replica method, the entropies are related to correlation functions of twist operators in a Euclidean BCFT. These correlations functions can be expanded in various channels involving intermediate bulk or boundary operators. Under certain sparseness conditions on the spectrum and OPE coefficients of bulk and boundary operators, we show that the twist correlators are dominated by the vacuum block in a single channel, with the relevant channel depending on the position of the twists. These transitions between channels lead to the holographically observed phase transitions in entropies.

KeYwords: Conformal Field Theory, AdS-CFT Correspondence, Black Holes in String Theory

ArXiv EPrint: 2004.13088 


\section{Contents}

1 Introduction 1

2 Review of boundary conformal field theory 4

3 Holographic BCFT entanglement entropies $\quad 9$

3.1 Holographic BCFTs 9

3.2 Entanglement entropies for holographic BCFTs 9

$\begin{array}{lll}\text { 3.2.1 } & \text { BCFT vacuum state on a half space } & 10\end{array}$

3.2.2 Entanglement entropy for boundary states $\left|b, \tau_{0}\right\rangle \quad 12$

3.2.3 Entanglement entropy in the thermofield double state of two BCFTs 14

4 BCFT calculation of entanglement entropies $\quad 14$

$\begin{array}{lll}4.1 & \text { Entanglement entropy from correlation functions of twist operators } & 15\end{array}$

$\begin{array}{lll}4.2 & \text { Two-point function of twist operators on a half-space } & 18\end{array}$

$\begin{array}{lll}4.3 & \text { Rényi entropy } & 20\end{array}$

4.4 BCFT requirements for vacuum block dominance 22

$\begin{array}{lll}4.4 .1 & \text { Boundary channel } & 22\end{array}$

$\begin{array}{ll}\text { 4.4.2 Bulk channel } & 24\end{array}$

4.5 Constraints on holographic BCFTs 24

4.5.1 Constraints on the BCFT base theory 25

$\begin{array}{lll}4.6 & \text { Black hole applications } & 26\end{array}$

5 Multiple intervals $\quad 28$

5.1 Holographic results for multiple intervals 28

$\begin{array}{lll}5.2 & \text { BCFT calculation for multiple intervals } & 29\end{array}$

6 Replica calculation in the gravity picture 31

$\begin{array}{lll}7 & \text { Discussion } & 34\end{array}$

A BCFT two-point functions from Virasoro conformal blocks 36

B Boundary operator expansion for twist operators 39

C Monodromy method 40

\section{Introduction}

In this note, we discuss the direct CFT calculation of entanglement and Rényi entropies for an interval (or collection of intervals) in some related 1+1-dimensional systems:

1. The vacuum state of a boundary conformal field theory (BCFT) on a half-space.

2. The state of a CFT on a circle produced by a Euclidean path integral with a boundary in the Euclidean past.

3. A pair of BCFTs in a thermofield double state. 
For holographic examples of these systems, calculations making use of the Ryu-Takayanagi (RT) formula [1] suggest that the entanglement entropy can undergo phase transitions related to a change in topology of the RT surface (for examples, see figures 1, 4 and 5). In this paper, we show that these holographic results can be reproduced with direct BCFT calculations by assuming large central charge and certain conditions on the CFT spectrum and OPE data. In particular, we show that the assumption of vacuum block dominance for BCFT correlators of twist operators is equivalent to a simple holographic prescription $[2,3]$ for the duals of BCFTs where the CFT boundary extends into the bulk as a purely gravitational end-of-the-world (ETW) brane with tension related to the boundary entropy of the BCFT (as suggested by [3]). ${ }^{1}$

This simple holographic prescription with a purely gravitational ETW brane is not expected to be valid universally for holographic BCFTs. In many cases, we can have nonvanishing one-point functions for light scalar operators in the BCFT. These translate to back-reacting scalar fields in the dual geometry that we can think of as being sourced by the ETW brane. In these cases with back-reacting scalars, the entanglement entropy for an interval in the phase corresponding to a connected RT surface (e.g. figure 1, top left) is a complicated function of the interval size and location and is not expected to be reproduced by the vacuum contribution in some channel.

On the other hand, the entanglement entropy in the phase corresponding to a disconnected RT surface is still simple and universal (see equation (3.8)) and reproduced by the vacuum block contribution to the boundary channel. Thus, while vacuum block dominance is not expected to hold universally for holographic BCFTs, the gravity results indicate that it does hold in this phase. This suggests a particular sparseness condition (4.39) that should hold for the spectrum of any holographic BCFT.

Probing black hole interiors. Our results have various applications to the physics of black hole interiors:

- In [5], it was argued that CFT states of type 2 above correspond to black hole microstates with a specific behind-the-horizon region whose geometry can be deduced by a standard holographic gravity calculation. Holographic calculations using the Ryu-Takayanagi (RT) formula [1] suggested that in many cases, the black hole interior can be probed via the entanglement entropy of sufficiently large subsystems of the CFT. Our direct CFT calculations confirm this expectation, precisely matching the results of [5].

- In [6], a pair of BCFTs in the thermofield double state was proposed as a microscopic model of a black hole in equilibrium with its Hawking radiation, following [7-9]. The RT surface for a fixed portion of the radiation system exhibits a phase transition as a function of time as the black hole and radiation system interact. After the transition, the entanglement wedge of this subsystem includes a portion of the black hole interior, suggesting that the black hole has transferred information about its interior to the radiation system through interaction. Our CFT calculations directly confirm the behavior of entanglement entropy surmised from the holographic calculation.

\footnotetext{
${ }^{1}$ The philosophy of reproducing gravitational results from vacuum block dominance goes by the amusing name "It from Id" [4].
} 

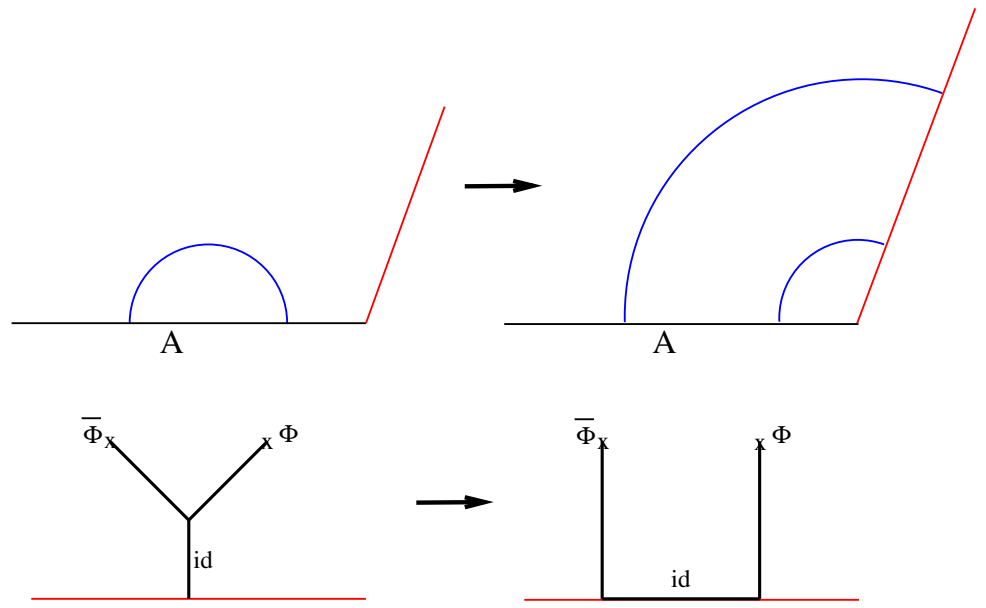

Figure 1. Top: transition in the RT surface from connected to disconnected topology; in black hole applications, the latter is associated with an entanglement wedge that includes the black hole interior. Bottom: BCFT interpretation in terms of the two-point function of twist operators used to compute entanglement entropy via the replica method. Phase transition comes from a switch of dominance between the identity block in a bulk channel to the identity block in a boundary channel.

BCFT methods. The analysis in this paper parallels the computation of Rényi entropies for multiple intervals in a CFT carried out by Hartman [10]. The CFT calculation makes use of the replica method, where we analytically continue the Rényi entropies to find the entanglement entropy. Rényi entropies are computed from the CFT path integral on a multi-sheeted surface, or equivalently, from correlation functions of twist operators in replicated version of the original BCFT.

These correlation functions can be expressed in terms of the basic BCFT data using operator product expansions. ${ }^{2}$ Different ways of performing this expansion (different "channels") give the same result, but at large $c$, and under certain conditions on the CFT spectrum, the leading term in a $1 / c$ expansion of the full result can be reproduced by truncating a single channel to include only intermediate states descending from the vacuum. Depending on the locations of the twist operators, this dominant channel can change, leading to a phase transitions in the entanglement entropy. The basic picture is illustrated in figure 1.

A running theme is the use of the doubling trick, and corresponding Ward identities, to simplify the kinematics of the BCFT. This relates the kinematics of BCFT $n$-point correlation functions on a half-plane to those of $2 n$-point correlation functions in a chiral CFT on the full plane. This applies not only to the single interval case, where a twopoint function in the BCFT is found to possess the same kinematics as a chiral four-point function, but to the multiple interval case, where the doubling trick can be applied to the monodromy method for computing conformal blocks.

Replica gravity calculation. Faulkner [11] simultaneously obtained the results in [10] from the gravity perspective. Instead of calculating replica partition functions using large

\footnotetext{
${ }^{2}$ Here, we have a bulk OPE, a bulk-boundary OE, and a boundary OPE.
} 
central charge CFT methods, the partition functions were calculated using gravitational path integrals in the dual Euclidean replica saddle geometries. We will see that Faulkner's calculation can be carried over directly to the calculation BCFT entanglement entropies. There, phase transitions in holographic entanglement entropy are mirrored by transitions in the dominant saddle contributing to the gravitational path integral. In the phase where the entanglement wedge of the radiation system includes the black hole interior, we can see explicitly from the Faulkner calculation that the relevant saddle is characterized by "replica wormholes", as suggested recently in [6, 12, 13].

Outline. In section 2 below, we provide some basic background on BCFTs and recall various results that we will need for our calculation. In section 3, we recall the properties of gravity duals for holographic BCFTs and the holographic calculation of entanglement entropy in the various examples mentioned above. In section 4, we present our main results, the direct (B)CFT calculation of entanglement and Renyi entropies, and an analysis of the conditions on the spectum and OPE coefficients necessary to reproduce the gravity predictions. In section 5, we generalize our BCFT results to the case of multiple intervals. Section 6 discusses the replica calculation of Renyi entropies on the gravity side and the relevance of replica wormholes. We finish with a discussion in section 7 .

\section{Review of boundary conformal field theory}

In this section, we start with a brief review of boundary conformal field theories. ${ }^{3}$

Given a CFT, we can define the theory on a manifold with boundary by making a choice of boundary conditions for the fields, and possibly adding boundary degrees of freedom coupled to the bulk CFT fields. For the theory defined on half of $\mathbb{R}^{d}$ or $\mathbb{R}^{d-1,1}$ (e.g. the region $x \geq 0$ for some spatial coordinate $x$ ), certain choices of the boundary physics give a theory that preserves the subset of the global conformal group mapping the half-space to itself, $\mathrm{SO}(d, 1) \subset \mathrm{SO}(d+1,1)$ for the Euclidean case. ${ }^{4}$ These choices define a boundary conformal field theory (BCFT). ${ }^{5}$ There are typically many choices of conformally invariant boundary condition for a given bulk CFT. We label the choice by an index $b$.

In this paper, we focus on BCFTs defined starting from two-dimensional conformal field theories. In this case, there is a natural boundary analog of the central charge, known as the boundary entropy $\log g_{b}[20]$. This may be defined by considering the CFT on a half-space $x \geq 0$ with boundary condition $b$ at $x=0$. As we review in section 3.1 below, the entanglement entropy of an interval $[0, L]$ including the boundary is

$$
S=\frac{c}{6} \log \frac{2 L}{\epsilon}+\log g_{b} .
$$

Thus, the boundary entropy gives a boundary contribution to the entanglement entropy. The quantity $g_{b}$ is also equal to the (regulated) partition function for the CFT on a disk with boundary condition $b$.

\footnotetext{
${ }^{3}$ For more detailed reviews of BCFTs, see e.g. [14-19].

${ }^{4}$ More generally, there can be boundary RG flows between such theories.

${ }^{5}$ We can also consider the same theory with different boundary geometries; in this paper, we will only consider geometries that can be mapped to a half-space via a conformal transformation.
} 
Boundary states. For any BCFT, there is a natural family of states $\left|b, \tau_{0}\right\rangle$ that we can associate to the parent CFT defined on a unit circle [21]. The wavefunctional $\left\langle\phi_{0} \mid b, \tau_{0}\right\rangle$ is defined as the Euclidean path integral for the CFT on a cylinder of height $\tau_{0}$, with boundary condition $b$ at Euclidean time $-\tau_{0}$ and CFT field configuration $\phi_{0}$ at $\tau=0 .{ }^{6}$ We can formally define a boundary state $|b\rangle$ associated with boundary condition $b$ via

$$
|b\rangle=\left|b, \tau_{0} \rightarrow 0\right\rangle
$$

In terms of $|b\rangle$, we have

$$
\left|b, \tau_{0}\right\rangle=e^{-\tau_{0} H}|b\rangle
$$

since adding $\delta \tau$ to the height of the cylinder corresponds to acting on our state with Euclidean time evolution $e^{-\delta \tau H}$. The boundary state itself has infinite energy expectation value, but the Euclidean evolution used to define $\left|b, \tau_{0}\right\rangle$ suppresses the high-energy components so that $\left|b, \tau_{0}\right\rangle$ is a finite energy state. ${ }^{7}$ In general, this state is time-dependent.

The overlap of the boundary state $|b\rangle$ with the vacuum state is computed via the path integral on a semi-infinite cylinder. This can be mapped to the disk via a conformal transformation, so the result is the disk partition function:

$$
\langle 0 \mid b\rangle=g_{b}
$$

Boundary operators. In addition to the usual CFT bulk operators, a BCFT has a spectrum of local boundary operators $\hat{O}_{J}(x)$, each with a dimension $\hat{\Delta}_{J}$. Via the usual radial quantization (taking the origin to be a point on the boundary), these may be understood to be in one-to-one correspondence with the states of the BCFT on an interval with the chosen boundary condition at each end. The boundary operator dimension is equal to the energy of the corresponding state on the strip.

Symmetries and correlators. A two-dimensional BCFT defined on the upper-half plane (UHP) preserves one copy of the Virasoro symmetry algebra, corresponding to transformations

$$
\delta z=\epsilon(z) \quad \delta \bar{z}=\bar{\epsilon}(\bar{z}) \quad \bar{\epsilon}(\bar{z})=\epsilon\left(\bar{z}^{*}\right)
$$

that map the boundary to itself. ${ }^{8}$ These correspond to a set of generators

$$
\tilde{L}_{n}=L_{n}+\bar{L}_{n}
$$

\footnotetext{
${ }^{6}$ With this definition, the norm of the states is not equal to 1.

${ }^{7}$ This is a version of the global quench considered in the condensed matter literature [19], but we have compactified the space on which the CFT is defined.

${ }^{8}$ Here, we recall that it is standard to treat $z$ and $\bar{z}$ as independent coordinates and consider a complexified version of the symmetry algebra for which the infinitesimal transformations are $\delta z=\epsilon(z)$ and $\delta \bar{z}=\bar{\epsilon}(\bar{z})$. The non-complexified transformations correspond to taking $\bar{\epsilon}(\bar{z})=\epsilon\left(\bar{z}^{*}\right)$ with $\epsilon(x)$ real for real $x$, or $\bar{\epsilon}(\bar{z})=-\epsilon\left(\bar{z}^{*}\right)$ with $\epsilon(x)$ pure imaginary for real $x$. Of these, the first set preserves the upper half plane, acting explicitly as $\delta x=(\epsilon(x+i y)+\epsilon(x-i y)) / 2, \delta y=-i(\epsilon(x+i y)-\epsilon(x-i y)) / 2$ on the physical coordinates.
} 
In this case, the conformal Ward identity becomes

$$
\begin{aligned}
\left\langle\tilde{T}(z) \prod_{i}\right. & \left.\mathcal{O}_{h_{i} \bar{h}_{i}}\left(z_{i}, \bar{z}_{i}\right)\right\rangle \\
& =\sum_{i}\left(\frac{h_{i}}{\left(z-z_{i}\right)^{2}}+\frac{1}{z-z_{i}} \frac{\partial}{\partial z_{i}}+\frac{\bar{h}_{i}}{\left(\bar{z}-\bar{z}_{i}\right)^{2}}+\frac{1}{\bar{z}-\bar{z}_{i}} \frac{\partial}{\partial \bar{z}_{i}}\right)\left\langle\prod_{i} \mathcal{O}_{h_{i} \bar{h}_{i}}\left(z_{i}, \bar{z}_{i}\right)\right\rangle,
\end{aligned}
$$

where $\tilde{T}(z)=\sum_{n} z^{-n-2} \tilde{L}_{n}$.

The Virasoro symmetry algebra of a BCFT is thus the same as that of a chiral CFT on the whole plane. A consequence is that the kinematics (i.e. the functional form of correlators given the operator dimensions) of the BCFT in the UHP is directly related to that of a chiral CFT on the whole plane. Correlators

$$
\left\langle\mathcal{O}_{h_{1} \bar{h}_{1}}\left(z_{1}, \bar{z}_{1}\right) \cdots \mathcal{O}_{h_{n} \bar{h}_{n}}\left(z_{n}, \bar{z}_{n}\right)\right\rangle_{\mathrm{UHP}}^{b}
$$

of bulk CFT operators $\mathcal{O}_{h_{k}} \bar{h}_{k}$ with conformal weights $\left(h_{k}, \bar{h}_{k}\right)$ in the UHP are constrained to have the same functional form as chiral CFT correlators

$$
\left\langle\mathcal{O}_{h_{1}}\left(z_{1}\right) \cdots \mathcal{O}_{h_{n}}\left(z_{n}\right) \mathcal{O}_{\bar{h}_{1}}\left(\bar{z}_{1}\right) \cdots \mathcal{O}_{\bar{h}_{n}}\left(\bar{z}_{n}\right)\right\rangle
$$

of fields $\mathcal{O}_{h_{k}}$ and $\mathcal{O}_{\bar{h}_{k}}$ with chiral weights $h_{k}$ and $\bar{h}_{k}$ respectively. ${ }^{9}$ More generally, we can include boundary operators $\hat{\mathcal{O}}_{\hat{\Delta}_{I}}\left(x_{I}\right)$ in (2.7), where $x_{I}$ is real. In this case, the functional form is reproduced by adding chiral operators with $h_{I}=\hat{\Delta}_{I}$ at $z=x_{I}$ to the chiral correlator (2.8). See [22] for a more complete discussion of this constraint, often referred to as the "doubling trick".

We will later make use of this kinematic equivalence to relate conformal blocks for a BCFT on the UHP to chiral conformal blocks on the entire plane.

Bulk one-point functions. The doubling trick implies that a primary operator with weights $(h, \bar{h})$ is kinematically allowed to have a nonvanishing one-point function if $h=\bar{h}$ (i.e. for a scalar primary). In this case, the one-point function $\left\langle\mathcal{O}_{h, h}(z, \bar{z})\right\rangle_{\mathrm{UHP}}^{b}$ is constrained to have the same form as a chiral two-point function $\left\langle\mathcal{O}_{h}(z) \overline{\mathcal{O}}_{h}\left(z^{*}\right)\right\rangle$, so we have

$$
\left\langle\mathcal{O}_{h, \bar{h}}(z, \bar{z})\right\rangle_{\mathrm{UHP}}^{b}=\frac{\mathcal{A}_{\mathcal{O}}^{b}}{\left|z-z^{*}\right|^{2 h}}=\frac{\mathcal{A}_{\mathcal{O}}^{b}}{|2 y|^{\Delta_{\mathcal{O}}}} .
$$

where we take $z=x+i y$ here and below. Once the normalization of the operators is fixed by choosing the normalization of the two-point function in the parent CFT, the coefficient $\mathcal{A}_{\mathcal{O}}^{b}$ in the one-point function is a physical parameter that depends in general on both the operator and the boundary condition.

Here and everywhere in this paper we will take the expectation value $\langle\cdot\rangle_{\mathrm{UHP}}^{b}$ to be normalized by the UHP partition function so that

$$
\langle\mathbf{1}\rangle_{\mathrm{UHP}}^{b}=1 \text {. }
$$

\footnotetext{
${ }^{9}$ Here, the original theory is defined on the slice where $\bar{z}=z^{*}$, so the operators $\mathcal{O}_{\bar{h}_{i}}\left(\bar{z}_{i}\right)$ live on the lower half-plane.
} 
Bulk-boundary two-point functions. The correlation function

$$
\left\langle\mathcal{O}_{i}(z, \bar{z}) \hat{\mathcal{O}}_{I}\left(x^{\prime}\right)\right\rangle_{\mathrm{UHP}}^{b}
$$

of bulk and boundary primary operators is constrained to have the functional form of a chiral three-point function

$$
\left\langle\mathcal{O}_{h_{i}}(z) \mathcal{O}_{I}\left(x^{\prime}\right) \mathcal{O}_{\bar{h}_{i}}(\bar{z})\right\rangle
$$

For a scalar operator $\mathcal{O}_{i}$, this gives

$$
\left\langle\mathcal{O}_{i}(z, \bar{z}) \hat{\mathcal{O}}_{I}\left(x^{\prime}\right)\right\rangle_{\mathrm{UHP}}^{b}=\frac{\mathcal{B}_{i I}^{b}}{(2 y)^{\Delta_{i}-\Delta_{I}}\left(y^{2}+\left(x-x^{\prime}\right)^{2}\right)^{\Delta_{I}}},
$$

where $\mathcal{B}_{i I}^{b}$ forms part of the basic data of our BCFT. Taking $\hat{\mathcal{O}}_{I}$ to be the identity operator, we have from the previous section that $\mathcal{B}_{i 1}^{b}=\mathcal{A}_{i}^{b}$.

Boundary operator expansion and OPEs. In the same way that a pair of bulk operators at separated points can be expanded as a series of local operators via the OPE, a bulk operator can be expanded in terms of boundary operators via a boundary operator expansion $(B O E) .{ }^{10}$ For a scalar primary operator, symmetries constrain the general form of this expansion to be

$$
\mathcal{O}_{i}(z, \bar{z})=\sum_{J} \frac{\mathcal{B}_{i}^{b J}}{(2 y)^{\Delta_{i}-\Delta_{I}}} \tilde{C}\left[y, \partial_{x}\right] \hat{\mathcal{O}}_{J}(x)=\sum_{J} \frac{\mathcal{B}_{i}^{b J}}{(2 y)^{\Delta_{i}-\Delta_{I}}} \hat{\mathcal{O}}_{J}(x)+\text { desc. }
$$

where the sum is over boundary primary operators. The differential operator $\tilde{C}$ determines the contribution of descendant operators and depends only on the conformal weights of $\mathcal{O}_{i}$ and $\hat{\mathcal{O}}_{J}$. The coefficients $\mathcal{B}_{i}^{b J}$ are related to the ones appearing in the bulk-boundary two-point function by raising the index with the metric $g_{I J}$ appearing in the boundary two-point function

$$
\left\langle\hat{\mathcal{O}}_{I}\left(x_{I}\right) \hat{\mathcal{O}}_{J}\left(x_{I}\right)\right\rangle=\frac{g_{I J}}{\left|x_{I}-x_{J}\right|^{2 \Delta_{I}}},
$$

though we will generally assume that we are working with a basis of boundary operators for which $g_{I J}=\delta_{I J}$.

Below, we will also make use of the ordinary OPE for bulk scalar operators, ${ }^{11}$

$$
\begin{aligned}
\mathcal{O}_{i}\left(z_{1}, \bar{z}_{1}\right) \mathcal{O}_{j}\left(z_{2}, \bar{z}_{2}\right) & =\sum_{k} \frac{\hat{\mathcal{C}}_{i j}^{k}}{\left|z_{1}-z_{2}\right|^{\Delta_{i}+\Delta_{j}-\Delta_{k}}} C_{\Delta_{i} \Delta_{j} ; \Delta_{k}}\left[z_{12}, \partial_{z}\right] \mathcal{O}_{k}\left(z_{2}, \bar{z}_{2}\right) \\
& =\sum_{k} \frac{\hat{\mathcal{C}}_{i j}^{k}}{\left|z_{1}-z_{2}\right|^{\Delta_{i}+\Delta_{j}-\Delta_{k}}} \mathcal{O}_{k}\left(z_{2}, \bar{z}_{2}\right)+\text { desc. }
\end{aligned}
$$

Finally, there is also an OPE for boundary fields, but we will not need this in our calculations below.

\footnotetext{
${ }^{10}$ This follows by the same logic of the state-operator mapping and OPE in a CFT. The state produced by a bulk operator can be mapped by an infinite dilation to a local operator at the origin on the boundary. And, as in the OPE, we choose to expand this local operator in terms of a basis of dilation eigenstates.

${ }^{11}$ To see that this should still be valid in the presence of a boundary, note that in a conformal frame where the upper-half-plane is mapped to the exterior of a circle surrounding the origin, the presence of the boundary is equivalent to the insertion of an operator at the origin (specifically, the operator associated with the state $\left|b, \tau_{0}\right\rangle$ described above).
} 
Two-point functions and conformal blocks. We now consider the bulk two-point function. Here, we restrict to scalar primary operators of equal dimension $\Delta$ since that is what we will need below. However, in general, bulk two-point functions in a BCFT can be non-vanishing for any conformal weights $\left(h_{1}, \bar{h}_{1}\right)$ and $\left(h_{2}, \bar{h}_{2}\right)$. We discuss the general case in detail in appendix A.

By the doubling trick, the BCFT two-point function

$$
\left\langle\mathcal{O}_{1}\left(z_{1}, \bar{z}_{1}\right) \mathcal{O}_{2}\left(z_{2}, \bar{z}_{2}\right)\right\rangle_{\mathrm{UHP}}^{b}
$$

of scalar operators with dimension $\Delta$ has the same functional form as a four-point function of chiral operators

$$
\left\langle\mathcal{O}_{1}\left(z_{1}\right) \mathcal{O}_{2}\left(z_{2}\right) \mathcal{O}_{3}\left(\bar{z}_{2}\right) \mathcal{O}_{4}\left(\bar{z}_{1}\right)\right\rangle
$$

where each operator has chiral weight $h=\Delta / 2$. Making use of (A.1) for the general form of such a correlator, we have that

$$
\left\langle\mathcal{O}_{1}\left(z_{1}, \bar{z}_{1}\right) \mathcal{O}_{2}\left(z_{2}, \bar{z}_{2}\right)\right\rangle_{\mathrm{UHP}}^{b}=\left[\frac{\eta}{4 y_{1} y_{2}}\right]^{\Delta} F(\eta)
$$

where $F(\eta)$ is some function of the cross-ratio

$$
\eta=\frac{\left(z_{1}-\bar{z}_{1}\right)\left(z_{2}-\bar{z}_{2}\right)}{\left(z_{1}-\bar{z}_{2}\right)\left(z_{2}-\bar{z}_{1}\right)}
$$

The function $F$ can be written more explicitly by making use of either the BOE or the bulk OPE for the operators in (2.19). Using the BOE for each operator in (2.19), the bulk two-point functions can be expressed as a sum of boundary two-point functions. In this way, the function $F(\eta)$ in (2.19) may be expressed as

$$
F(\eta)=\sum_{I} \mathcal{B}_{\mathcal{O}_{1}}^{b I} \mathcal{B}_{\mathcal{O}_{2} I}^{b} \mathcal{F}\left(c, \Delta_{I}, \Delta / 2 \mid \eta\right)
$$

where the sum is over boundary primary operators and $\mathcal{F}\left(c, \Delta_{I}, \Delta / 2 \mid \eta\right)$ gives the contribution of a single boundary primary operator and all of its Virasoro descendants. We show in appendix A that this function is the usual conformal block appearing in the expansion of a chiral four-point function of operators with equal conformal weight $h=\Delta / 2{ }^{12}$

We can alternatively use the bulk OPE to reduce the BCFT two-point function (2.19) to a sum of one-point functions. This leads to an alternative expression for $F(\eta)$,

$$
F(\eta)=\sum_{i} \mathcal{C}_{\mathcal{O}_{1} \mathcal{O}_{2}}^{i} \mathcal{A}_{i}^{b} \mathcal{F}\left(c, \Delta_{I}, \Delta / 2 \mid 1-\eta\right)
$$

Here, $\mathcal{F}$ is the same chiral conformal block as in (2.21), as we show in appendix A. The equivalence of the expressions (2.21) and (2.22) is a BCFT version of the usual crossing symmetry constraints; in this case, we have a relation between bulk OPE coefficients and boundary operator expansion coefficients.

\footnotetext{
${ }^{12}$ In general, the conformal block depends on four external weights; here and below, we will use the shorthand $\mathcal{F}\left(c, h_{\text {int }}, h \mid \eta\right) \equiv \mathcal{F}\left(c, h_{\text {int }},[h, h, h, h] \mid \eta\right)$ where the latter is the general expression for the chiral conformal block used in appendix A.
} 


\section{$3 \quad$ Holographic BCFT entanglement entropies}

In this section, we review the holographic calculation of entanglement entropies for subsystems of BCFTs with gravitational duals, or for states of holographic CFTs defined via Euclidean BCFT path integrals. These are the results that we will try to understand via direct CFT calculations in the next section.

\subsection{Holographic BCFTs}

Certain BCFTs have a dual gravitational description. These correspond to holographic CFTs defined on a space $M$ with boundary $\partial M$, and a boundary condition perhaps obeying additional constraints so that the theory remains holographic. The dual geometries are asymptotically AdS with boundary geometry $M$, but the bulk physics associated with $\partial M$ can be different depending on the choice of boundary condition.

For a $d$-dimensional CFT, we can have an effective "bottom up" description of the gravity dual as a $(d+1)$-dimensional asypmtotically AdS spacetime with an end-of-theworld (ETW) brane extending from $\partial M[2,3,23,24]$. However, in "top down" microscopic examples (see for instance [25-30]), the dual can be a smooth higher-dimensional geometry. In this case, the ETW brane in the lower-dimensional description represents the smooth degeneration of an internal dimension.

The simplest possible gravitational dual has an ETW brane coupling only to the bulk metric field. Its action is taken to include a boundary cosmological constant (interpreted as the brane tension) and a Gibbons-Hawking term involving the trace of the extrinsic curvature. The details of the action and equation of motion, and all the solutions that we will require in this paper, may be found in [5]. A more general ansatz is an ETW brane action with coupling to additional bulk fields, e.g. light scalars.

\subsection{Entanglement entropies for holographic BCFTs}

We can use the Ryu-Takayanagi (RT) formula [1] to holographically calculate the entanglement entropy for spatial subsystems. As usual, the entropy (at leading order in the $1 / c$ expansion) is given as

$$
S_{A}=\frac{1}{4 G} \operatorname{Area}(\tilde{A}),
$$

where $\tilde{A}$ is the minimal area extremal surface in the dual geometry homologous to the boundary region $A$.

A new feature of entanglement entropy for holographic BCFTs is that the RT surfaces can end on the ETW brane [3]. Here, we should keep in mind that the ETW brane itself represents a part of the bulk geometry. The homology condition says that the RT surface $\mathcal{X}_{A}$ for a region $A$ on the boundary, together with the region $A$ itself, should be the boundary of a region $\Xi_{A}$ of the bulk spacetime: $\partial \Xi_{A}=A \cup \mathcal{X}_{A}$. But when applying this condition, the ETW brane should be considered as part of this bulk spacetime region $\Xi_{A}$, rather than an additional contribution to the boundary. As a result, we can have a disconnected RT surface for a connected boundary region, as shown in figure 3 . 


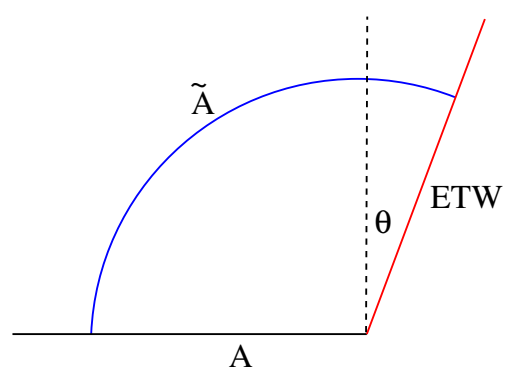

Figure 2. Holographic calculation of entanglement entropy for an interval $A$ containing the boundary. The RT surface $\tilde{A}$ sits at a fixed location on the $\mathrm{AdS}_{2}$ fibers of the dual geometry. Here $A$ is homologous to $\tilde{A}$ since the ETW brane represents a smooth part of full microscopic geometry.

\subsubsection{BCFT vacuum state on a half space}

As an example, consider the vacuum state of a two-dimensional BCFT on a half-space $x>0$. Here, the $\mathrm{SO}(1,2)$ symmetry preserved by the BCFT should be reflected in the dual geometry. Generally, this gives a warped product of $\mathrm{AdS}_{2}$ and an internal space, such that the full geometry has an asymptotic region that is locally $\mathrm{AdS}_{3}$ times some internal space, with boundary geometry equal to the half-space on which the CFT lives. In general, we can write the metric as

$$
\mathrm{d} s_{\mathcal{M}}^{2}=\ell_{\text {AdS }}^{2}\left[\hat{g}_{i j}(\mu) \mathrm{d} \mu_{i} \mathrm{~d} \mu_{j}+\frac{f(\mu)}{z^{2}}\left(\mathrm{~d} z^{2}-\mathrm{d} t^{2}\right)\right] .
$$

Microscopic solutions of this type were constructed in [25, 26].

We can also give a lower dimensional description (at least in the vicinity of the boundary), where we reduce on the internal space so that the internal metric is represented via scalars and vectors. In this case, we can write

$$
\mathrm{d} s_{\mathcal{M}}^{2}=\ell_{\text {AdS }}^{2}\left[\mathrm{~d} \mu^{2}+\frac{f(\mu)}{z^{2}}\left(\mathrm{~d} z^{2}-\mathrm{d} t^{2}\right)\right],
$$

where $f(\mu) \rightarrow \cosh ^{2}\left(\mu / \ell_{\text {AdS }}\right)$ as we approach the asymptotic boundary at $\mu=-\infty$ so that the metric is asymptotically $\mathrm{AdS}_{3}$. In general, the scalar fields in the geometry can be functions of the coordinate $\mu$.

In the simplest effective bulk theory, there is an ETW brane with stress-energy tensor $8 \pi G T_{a b}=-T g_{a b} / \ell_{\text {AdS }}[2,3]$, and bulk geometry pure AdS, with $f(\mu)=\cosh ^{2}\left(\mu / \ell_{\text {AdS }}\right)$. The brane sits at $\mu_{\max }=\operatorname{arctanh}(T)$. Here, the coordinate $\mu$ is related to the angular coordinate $\theta$ in a polar-coordinate description of Poincaré-AdS by $1 / \cos (\theta)=\cosh (\mu)$, so the brane goes into the bulk at a constant angle $\theta=\arcsin (T)$, as shown in figure 2 .

Entanglement entropy for an interval including the boundary. We now consider the entanglement entropy for an interval in the half-space. In the case of an interval $[0, L]$ containing the boundary, we expect the universal form (2.1) for the entanglement entropy. In the holographic calculation with the general metric (3.2), the RT surface sits at a constant position on the $\mathrm{AdS}_{2}$ fiber, so the entanglement entropy is

$$
S=\frac{1}{4 G} \int_{z>\epsilon} \mathrm{d}^{d+1} x \sqrt{\hat{g}},
$$




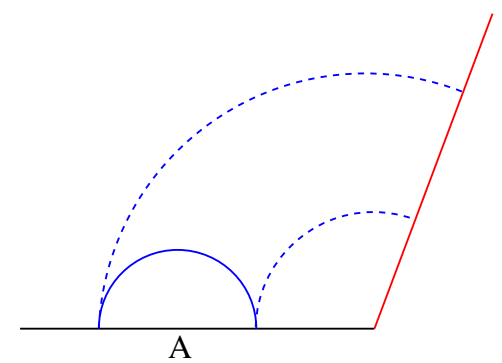

Figure 3. Holographic calculation of entanglement entropy for an interval $A$ away from the boundary. The RT surface has two possible topologies, a connected (solid curve) and disconnected (dashed curves).

where $z$ is the Fefferman-Graham radial coordinate and $d$ is the dimension of the internal space. We can regulate this by subtracting off half the area of the entangling surface of an interval of length $2 L$ in vacuum AdS, so

$$
S=\left[\frac{\ell_{\mathrm{AdS}}^{d+1}}{4 G} \int_{z>\epsilon} \mathrm{d}^{d} \mu_{i} \sqrt{\hat{g}}-\frac{\ell_{\mathrm{AdS}}^{d+1}}{4 G} \int_{z>\epsilon, x>0} \mathrm{~d}^{d} x \sqrt{\hat{g}_{\mathrm{AdS}}}\right]+\frac{\ell_{\mathrm{AdS}}^{d+1}}{4 G} \int_{z>\epsilon, x>0} \mathrm{~d}^{d} x \sqrt{\hat{g}_{\mathrm{AdS}}},
$$

where $\hat{g}_{\text {AdS }}$ is the metric for pure AdS. In this expression, the term in square brackets has a finite limit as $\epsilon \rightarrow 0$, independent of $L$, while the second term gives $\frac{c}{6} \log \left(\frac{2 L}{\epsilon}\right)$. Thus, we reproduce (2.1), with the identification

$$
\log g_{b}=\lim _{\epsilon \rightarrow 0}\left[\frac{\ell_{\mathrm{AdS}}^{d+1}}{4 G} \int_{z>\epsilon} \mathrm{d}^{d} x \sqrt{\hat{g}}-\frac{\ell_{\mathrm{AdS}}^{d+1}}{4 G} \int_{z>\epsilon, x>0} \mathrm{~d}^{d} x \sqrt{\hat{g}_{\mathrm{AdS}}}\right] .
$$

As an example, with a constant tension ETW brane, we have

$$
\log g_{b}=\frac{\ell_{\mathrm{AdS}}}{4 G} \int_{0}^{\mu_{\max }} \mathrm{d} \mu=\frac{\ell_{\mathrm{AdS}}}{4 G} \operatorname{arctanh}(T)=\frac{c}{6} \operatorname{arctanh}(T) .
$$

This is the result of Takayanagi [3] relating the boundary entropy to brane tension.

Entanglement entropy for an interval away from the boundary. Now consider the holographic calculation of entanglement entropy for an interval $\left[x_{1}, x_{2}\right]$ away from the boundary. In general, the CFT result for this entanglement entropy does not have a universal form.

In the holographic calculation, we can have a phase transition between two different possible RT surface topologies: a connected RT surface or a disconnected surface with both components ending on the ETW brane. The two topologies are shown in figure $3 .{ }^{13}$

Let us consider these phases in more detail. In the disconnected case, the RT surface computing the entanglement entropy of an interval $\left[x_{1}, x_{2}\right]$ is the union of the RT surfaces associated with $\left[0, x_{1}\right]$ and $\left[0, x_{2}\right]$. Thus, to leading order in large $N$, we have that

$$
S_{\left[x_{1}, x_{2}\right]}^{\mathrm{disc}}=S_{\left[0, x_{1}\right]}+S_{\left[0, x_{2}\right]}=\frac{c}{6} \log \left(\frac{2 x_{1}}{\epsilon}\right)+\frac{c}{6} \log \left(\frac{2 x_{2}}{\epsilon}\right)+2 \log g_{b} .
$$

\footnotetext{
${ }^{13}$ This transition in holographic entanglement entropy was discussed in detail for $\mathrm{AdS}_{4} / \mathrm{BCFT}_{3}$ and higher dimensions in [31]. We thank an anonymous reviewer for pointing this out.
} 
This result makes use only of the disconnected topology of the RT surface, so is a universal result for the disconnected phase in any holographic theory.

In the connected phase (expected to apply when the interval is sufficiently far from the CFT boundary), there is in general no simple universal result for the entanglement entropy. We need to find an RT surface in the dual geometry, and the calculation of this surface will depend on the details of the metric $\hat{g}$ appearing in (3.2).

For certain boundary conditions, it may be that the dual gravitational theory is welldescribed by an ETW brane with only gravitational couplings. In this case, the dual geometry is locally $\mathrm{AdS}_{3}$, and the calculation of entanglement entropy for the interval will be the same as the holographic calculation of vacuum entanglement entropy for the same interval in the CFT without a boundary. Thus, we have

$$
S_{\left[x_{1}, x_{2}\right]}^{\mathrm{conn}}=\frac{c}{3} \log \left(\frac{x_{2}-x_{1}}{\epsilon}\right) .
$$

Below, we will try to understand what conditions must be satisfied in the BCFT in order that this result is correct.

In cases where (3.8) and (3.9) give the correct results for the two possible RT-surface topologies, the actual entanglement entropy will be computed by taking the minimum of these two results. We find that the disconnected surface gives the correct result for the entanglement entropy when

$$
\log \left[\frac{1}{2}\left(\sqrt{\frac{x_{2}}{x_{1}}}-\sqrt{\frac{x_{1}}{x_{2}}}\right)\right]>\frac{6 \log g_{b}}{c},
$$

so that for a fixed interval size, we have a phase transition as the location of the interval relative to the boundary is varied.

In the more general case where the bulk geometry is not locally AdS, there is no explicit result for the entanglement entropy in the connected phase and (3.10) does not apply. However, we expect that the qualitative behavior of the entanglement entropy is similar, with a transition to the disconnected phase as the interval approaches the boundary. We can view this as a prediction for the behavior of entanglement entropy in holographic BCFTs. One of our main goals below will be to understand the existence of this transition via a direct CFT calculation.

Before turning to the machinery of CFTs, we review two closely related holographic calculations. In both cases, the dual geometry involves a black hole, and the transition in RT surfaces takes us between phases where the entanglement wedge of the CFT region under consideration does or does not include a portion of the black hole interior.

\subsubsection{Entanglement entropy for boundary states $\left|b, \tau_{0}\right\rangle$}

Consider a CFT on $S^{1}$, in the state $\left|b, \tau_{0}\right\rangle$ defined via the Euclidean path integral (2.3). We can consider the entanglement entropy for an interval of angular size $\Delta \theta$ in this state, at some fixed Lorentzian time. As described in [5, 32], for small enough $\tau_{0}$, this is a highenergy pure state of the CFT and the dual geometry is expected to be black hole. Assuming that the bulk effective gravitational theory for the BCFT involves a purely gravitational 

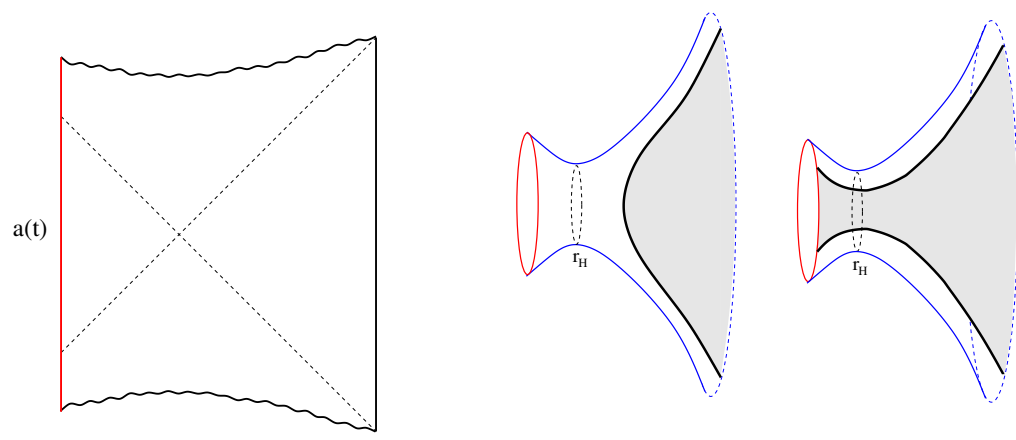

Figure 4. The dual geometry for $\left|b, \tau_{0}\right\rangle$ for sufficiently small $\tau_{0}$ is a portion of the maximallyextended AdS-Schwarzchild geometry, cut off by a spherically symmetric ETW brane. The pictures on the right show the spatial slice at $t=0$ and the connected and disconnected topologies for the RT surface corresponding to a large interval on the boundary circle.

ETW brane of tension $T$, it was shown in $[5,32]$ that the dual geometry for $T>0$ is a portion of the maximally-extended AdS-Schwarzchild geometry. The black hole interior terminates on a spherically-symetric ETW brane with a time-dependent radius, as shown in figure 4.

In this case, the geometry outside the horizon is pure AdS-Schwarzchild. In the connected phase, which dominates for small enough $\Delta \theta$, the RT surface lies entirely outside the horizon and gives a time-independent entanglement entropy

$$
S^{\text {conn }}=\frac{c}{3} \log \left[\frac{4 \tau_{0}}{\pi \epsilon} \sinh \left(\frac{\pi \Delta \theta}{4 \tau_{0}}\right)\right],
$$

where we take the circumference of the CFT circle to be 1 .

For small enough $\tau_{0}$, large-enough interval size $\Delta \theta$, and time $t$ sufficiently close to 0 (when the state is prepared) we also have a disconnected phase, where the RT surface is a union of two surfaces at fixed angular position that enter the horizon and terminate on the ETW brane. Here, we find that

$$
S^{\text {disc }}=\frac{c}{3} \log \left[\frac{4 \tau_{0}}{\epsilon \pi} \cosh \left(\frac{\pi t}{2 \tau_{0}}\right)\right]+2 \log g_{b} .
$$

This is smaller than the connected result (and thus represents the actual entanglement entropy) when

$$
\sinh \left(\frac{\pi \Delta \theta}{4 \tau_{0}}\right) \geq \cosh \left(\frac{\pi t}{2 \tau_{0}}\right) e^{\frac{6 \log g_{b}}{c}} .
$$

When this condition is satisfied, the entanglement wedge of the interval includes a portion of the black hole interior, and hence the entanglement entropy probes the interior geometry. For late times, the connected phase always dominates. This is consistent with the expectation that the state will thermalize, so that the entanglement entropy for a subsystem gives the thermal result. ${ }^{14}$

\footnotetext{
${ }^{14}$ This is similar to the behaviour of correlators in microscopic models of black hole collapse based on approximate global quenches of Vaidya type [4]. A phase transition in channel dominance leads to a shift in the gravitational saddle computing entanglement entropy, which in turn is responsible for maintaining unitarity. We thank Tarek Anous for discussion of this point.
} 


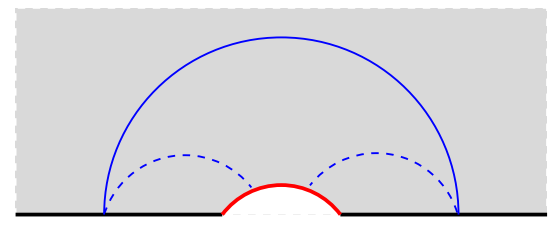

Figure 5. Dual geometry to the thermofield double state of two BCFTs, showing two possible topologies for the RT surface for the region corresponding to the union of points in either CFT at a distance larger than $x_{0}$ from the boundary.

\subsubsection{Entanglement entropy in the thermofield double state of two BCFTs}

In our final example, we take the thermofield double state of two BCFTs, each on a halfspace, and consider the entanglement entropy for the subsystem $A\left(x_{0}\right)$ consisting of the union of the regions $\left[x_{0}, \infty\right)$ in each CFT, as in figure 8d. In [6], following [9], it was argued that this system provides a model of a two-sided 2D black hole coupled to an auxiliary radiation system, where the Page time for the black hole is $t_{\text {Page }} \sim 6 \log \left(g_{b}\right) / c$.

While the simple observables in this system are time-independent, the holographic calculations in [6] revealed that the entanglement entropy for the subsystem $A\left(x_{0}\right)$ increases with time, then undergoes a phase transition. After this transition, the entanglement entropy is time-independent and the entanglement wedge of the radiation system includes a substantial portion of the black hole interior. The interpretation is that while no net energy is exchanged between the black hole and the radiation system, information from the black hole escapes into the radiation system until the radiation system contains enough of it to reconstruct the black hole interior.

The phase transition is seen most easily via a holographic calculation in the dual three-dimensional gravity picture. Here, we have a dynamical ETW brane that connects the two CFT boundaries, as shown in figure 5. At early times, the RT surface for $A\left(x_{0}\right)$ is connected and does not intersect the ETW brane. The entanglement entropy is

$$
S^{\text {conn }}=\frac{c}{3} \log \left(\frac{2}{\epsilon} \cosh t\right) .
$$

At late times, the RT surface is disconnected with components stretching from the boundary of each BCFT to the ETW brane. In this case, the entropy is

$$
S^{\text {disc }}=\frac{c}{3} \log \left(\frac{2}{\epsilon} \sinh x_{0}\right)+2 \log g_{b} .
$$

This is smallest (and thus gives the correct result) for $\cosh t>e^{\frac{6 \log g_{b}}{c}} \sinh x_{0}$.

In the next section, we will consider the direct CFT calculation of the entanglement entropies for the situations we have just described. As we explain in section 4.6, while the three calculations correspond to rather different physical scenarios, the underlying CFT calculation of the entanglement entropies is are directly related.

\section{BCFT calculation of entanglement entropies}

In this section, we move on to our central task: performing a direct CFT calculation of entanglement entropy for one or more intervals in the vacuum state of a BCFT on a half- 

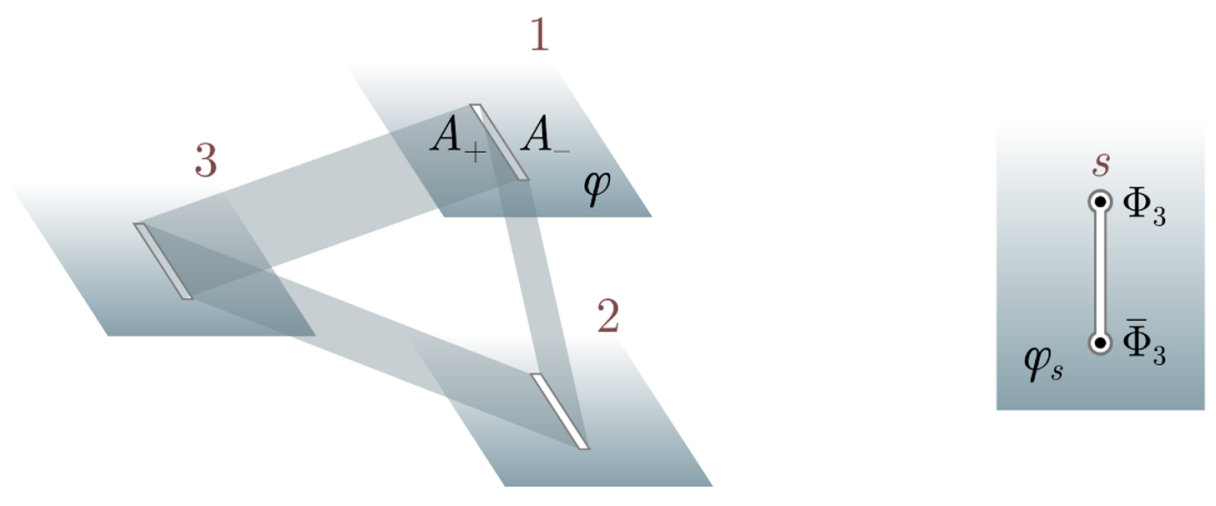

Figure 6. Left. Three-replica geometry, $\mathcal{R}_{3}$, with a local field $\varphi$. Right. Individual copies $s$, with boundary conditions for $\varphi_{i}$ implemented by twists $\Phi_{3}, \bar{\Phi}_{3}$.

line, for the thermofield double state of two BCFTs, or for the CFT state $\left|b, \tau_{0}\right\rangle$ generated by a Euclidean BCFT path integral. We will argue that with certain assumptions, we can directly reproduce the holographic results described in the previous section.

\subsection{Entanglement entropy from correlation functions of twist operators}

We begin by briefly recalling the CFT calculation of entanglement entropy (for more details, see [33]). We consider a CFT or BCFT on a spatial geometry $M$ in some state $|\Psi\rangle$, defined by a Euclidean path integral on a geometry $H$ with boundary $M$. We would like to calculate the entanglement entropy $S_{A}=-\operatorname{tr}\left(\rho_{A} \log \rho_{A}\right)$ for a region $A \subset M$.

The entanglement entropy can be obtained from a limit of $n$-Rényi entropies $S_{A}^{(n)}$ :

$$
S_{A}=\lim _{n \rightarrow 1} S_{A}^{(n)}, \quad S_{A}^{(n)}:=\frac{1}{1-n} \log \operatorname{Tr}\left[\rho_{A}^{n}\right] .
$$

The matrix elements $\left\langle\phi_{A}^{-}\left|\rho_{A}\right| \phi_{A}^{+}\right\rangle$are calculated from the path integral on a space $(\bar{H} H)_{A}$ formed from gluing two copies of $H$ along the complement of $A$ in $M,{ }^{15}$ where we set boundary conditions $\phi(x, \tau= \pm \epsilon)=\phi_{A}^{ \pm}$on either side of a cut $A$. The proper normalization is obtained by dividing by the same path integral without a cut along $A$.

The trace $\operatorname{Tr}\left[\rho_{A}^{n}\right]$ is then obtained by the path integral on a replica geometry $\mathcal{R}_{n}$ obtained by gluing $n$ copies of $(\bar{H} H)_{A}$ across the cut A, with the lower half of the cut on each copy glued to the upper half of the cut on the next copy, as shown in figure 6. Including the proper normalization in the path integral expression for the density matrix gives

$$
\operatorname{Tr}\left[\rho_{A}^{n}\right]=\frac{Z_{n}}{Z_{1}^{n}}
$$

where $Z_{n}$ is the partition function for the CFT on $\mathcal{R}_{n}$.

The ratio $Z_{n} / Z_{1}^{n}$ can be expressed as a correlation function of twist operators for a CFT/BCFT defined to be the product of $n$ copies of the original theory. A twist operator $\Phi_{n}(z)$ inserted at $z$ is defined via the path integral by inserting a branch cut ending at

\footnotetext{
${ }^{15}$ More precisely, the path integral corresponding to the second copy is the one associated with $\langle\Psi|$; any complex sources in the action should be conjugated.
} 

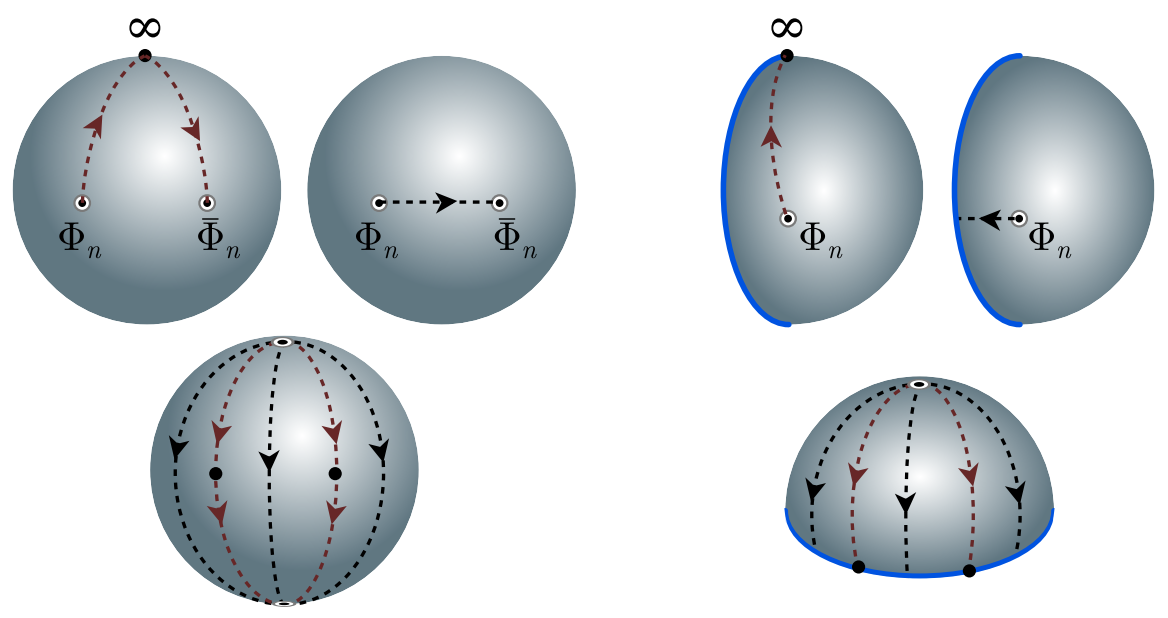

Figure 7. Left. Deforming the contour of the fundamental domain of $\mathcal{R}_{n}$ for a CFT. Right. Performing the equivalent deformation on $\mathcal{R}_{n}$ for a BCFT.

$z$, across which the fields in the $k$ th copy of the (B)CFT are identified with fields in the $(k+1)$-st copy as we move clockwise around the branch point. Similarly, an anti-twist operator $\bar{\Phi}_{n}(z)$ inserts a branch cut ending at $z$ across which fields in the $k$ th copy of the CFT/BCFT are identified with fields in the $(k-1)$-st copy as we move clockwise around the branch point.

In a CFT, every twist operator must come with an anti-twist operator, with the branch cut running between the two. For a BCFT, in contrast, we can have an unpaired twist operator, with the branch cut running between the operator insertion and the boundary. For both the CFT and BCFT, deforming the branch cut simply corresponds to changing the fundamental domain of the replica Riemann surface $\mathcal{R}_{n}$, as in figure 7 .

Two-point function of the twist operators in a CFT. The correlator $\left\langle\bar{\Phi}_{n}\left(x_{1}\right) \Phi_{n}\left(x_{2}\right)\right\rangle$ for the $n$-copy CFT on the real line with a branch cut running between $x_{1}$ and $x_{2}$ exactly computes the right-hand side of (4.2) for the case where $A$ is the interval $\left[x_{1}, x_{2}\right]$. The two point function takes a simple form, since as shown in [17], the twists fields $\Phi_{n}, \bar{\Phi}_{n}$ act like scalar primaries with scaling dimension

$$
d_{n}:=\frac{c}{12}\left(n-\frac{1}{n}\right)
$$

and weights $h_{n}=\hat{h}_{n}=d_{n} / 2$. Thus, we have

$$
\left\langle\bar{\Phi}_{n}\left(x_{1}\right) \Phi_{n}\left(x_{2}\right)\right\rangle \sim\left|x_{1}-x_{2}\right|^{-2 d_{n}},
$$

as we will derive again below.

To say more about the coefficient, we need to define the twist operators more precisely by specifying the behavior of the CFT at the branch points. As a specific regularization, we can consider instead the $n$-copy theory defined on a space obtained by removing a disk of radius $\epsilon$ centered at each branch point and placing boundary condition labelled by $a_{i}$ at 
the $i$ th resulting circular boundary [34]. ${ }^{16}$ The resulting path integral geometries for $Z_{n}$ (and $Z_{1}$ ) are then smooth. A conformal transformation

$$
z \mapsto i \log \left(\frac{z-x_{2}}{z-x_{1}}\right)
$$

maps the original plane to a cylinder defined by the complex plane with identification $z \sim z+2 \pi$, and the branch cut $\left[x_{1}, x_{2}\right]$ mapping to $\Re(z)=\pi$.

For small $\epsilon$, the boundaries surrounding the branch points map to $z= \pm i \log \left[\left(x_{2}-\right.\right.$ $\left.x_{1}\right) / \epsilon$ ] up to corrections of order $\epsilon$. Thus, the path integral geoemtry is a cylinder of length $\tau=2 \log \left[\left(x_{2}-x_{1}\right) / \epsilon\right]$, with boundary condition $a_{1}, a_{2}$ at the two ends. The replica geometry is defined by gluing $n$ copies of this cylinder along the vertical branch cut, so corresponds to a cylinder with circumference $2 \pi n$. We can write the path integral on this space using boundary states as ${ }^{17}$

$$
Z_{n}=\left\langle a_{2}\left|e^{-\frac{\tau}{2 \pi n} H}\right| a_{1}\right\rangle
$$

where $H$ is the Hamiltonian for the CFT on a circle of unit length. For large $\tau$, the operator inside approaches a projector to the vacuum state

$$
e^{-\frac{\tau}{2 \pi n} H} \rightarrow e^{-\frac{\tau}{2 \pi n} E_{0}}|0\rangle\langle 0|,
$$

where $E_{0}=-\pi c / 6$ is the vacuum energy for a CFT on a circle of unit length.

Thus, we get

$$
\left(Z_{n}\right)_{\epsilon \rightarrow 0}=\left(\frac{\left|x_{2}-x_{1}\right|}{\epsilon}\right)^{\frac{c}{6 n}}\left\langle a_{2} \mid 0\right\rangle\left\langle 0 \mid a_{1}\right\rangle
$$

Finally,

$$
\left\langle\bar{\Phi}_{n}\left(x_{1}\right) \Phi_{n}\left(x_{2}\right)\right\rangle=\frac{Z_{n}^{a, \epsilon}}{\left(Z_{1}^{a, \epsilon}\right)^{n}}=\left(\left\langle a_{2} \mid 0\right\rangle\left\langle 0 \mid a_{1}\right\rangle\right)^{(1-n)}\left(\frac{\left|x_{1}-x_{2}\right|}{\epsilon}\right)^{-2 d_{n}} .
$$

Making use of this in (4.1) and (4.2) gives the standard result for the entanglement entropy of an interval. With our original definition of $\epsilon$, we have $c / 3 \log (L / \epsilon)+\log g_{a_{1}}+\log g_{a_{2}}$, however, it will be convenient to take $a_{1}=a_{2}=a$ and absorb the last two terms here into the definition of $\epsilon$.

One-point function of the twist operator on a half space. In a BCFT, the twist operators also have a non-vanishing one-point function, related to the Rényi entropies for an interval $[0, x]$ in the vacuum state of the BCFT on a half-space $x \geq 0$. We can calculate this using the regularization defined above.

We will consider a BCFT on the UHP with boundary condition $b$ at $\Im(z)=0$, with the twist operator at $z_{1}=x_{1}+i y_{1}$ regulated by boundary condition $a$. The conformal transformation

$$
z \mapsto i \log \left(\frac{z-z_{1}^{*}}{z-z_{1}}\right)
$$

\footnotetext{
${ }^{16}$ It will be convenient for our discussion below to allow different boundary conditions to regulate the different twist operators, but generally we can choose the same one for each.

${ }^{17}$ We recall that the boundary state was defined using a circle of length 1 . Scaling the cylinder to have this circumference, the length becomes $\tau / 2 \pi n$.
} 
maps the upper half-plane to a cylinder defined by the complex plane with identification $z \sim z+2 \pi$, where the boundary along the real axis maps to the interval $[0,2 \pi]$ on the real axis. The circle of radius $\epsilon$ regulating the twist operator maps (in the limit of small $\epsilon$ ) to a second end of the cylinder at $\Im(z)=\log \left(2 y_{1} / \epsilon\right)$.

Thus, the one-point function is $Z_{n} /\left(Z_{1}\right)^{n}$, where $Z_{n}$ is the partition function on a cylinder of circumference $2 \pi n$ and height $\tau=\log \left(2 y_{1} / \epsilon\right)$. Using the second equation in (4.9), we have that

$$
\left\langle\Phi_{n}\left(z_{1}, \bar{z}_{n}\right)\right\rangle=\frac{Z_{n}}{\left(Z_{1}\right)^{n}}=(\langle a \mid 0\rangle\langle 0 \mid b\rangle)^{(1-n)}\left|\frac{2 y_{1}}{\epsilon}\right|^{-d_{n}},
$$

Interpreting the $\Re(z)$ direction as Euclidean time, this gives $\operatorname{tr}\left(\rho^{n}\right)$ for an interval $\left[0, y_{1}\right]$ in the vacuum state of a BCFT on a half-space. From (4.1), the entanglement entropy associated with this density matrix is

$$
S=\frac{c}{6} \log \left(\frac{2 y_{1}}{\epsilon}\right)+\log \left(g_{a}\right)+\log \left(g_{b}\right) .
$$

The term $\log \left(g_{a}\right)$ in the regulator can be absorbed by a redefinition of $\epsilon$ to give the result (2.1). ${ }^{18}$ On the other hand, boundary entropy term $\log \left(g_{b}\right)$ is physical. It is equal to the difference between the BCFT entanglement entropy and the half the entanglement entropy in the parent CFT for an interval of length $2 y_{1}$, with twist regularization fixed.

\subsection{Two-point function of twist operators on a half-space}

We are now ready for our main calculation. We consider the correlator on the UHP of a twist operator at $z_{1}$ and an anti-twist operator at $z_{2}$. As discussed in section 2 , we can express the two-point function here either as a sum of bulk one-point functions (the bulk channel), or as a sum of boundary two-point functions (the boundary channel), using the bulk OPE or the BOE respectively. We now consider these expressions explicitly.

Boundary channel for the two-point function. The boundary channel for the BCFT two-point function is obtained by first expanding each operator using the BOE, so that the bulk two-point function becomes a sum of boundary two-point functions. The contribution of two-point functions involving all the operators in a multiplet of the Virasoro symmetry sums to a conformal block. Using the general result (2.19) with (2.21), we find

$$
\left\langle\Phi_{n}\left(z_{1}, \bar{z}_{1}\right) \bar{\Phi}_{n}\left(z_{2}, \bar{z}_{2}\right)\right\rangle_{\mathrm{UHP}}^{b}=\left[\frac{\eta}{4 y_{1} y_{2}}\right]^{d_{n}} \sum_{I} \mathcal{B}_{\Phi I}^{b} \mathcal{B}_{\bar{\Phi} I}^{b} \mathcal{F}\left(c, \Delta_{I}, \frac{d_{n}}{2} \mid \eta\right)
$$

where

$$
\eta=\frac{\left(z_{1}-\bar{z}_{1}\right)\left(z_{2}-\bar{z}_{2}\right)}{\left(z_{1}-\bar{z}_{2}\right)\left(z_{2}-\bar{z}_{1}\right)}
$$

and where $I$ indexes untwisted boundary operators in the $n$-fold product theory. As we review in appendix $\mathrm{B}$, the $\mathrm{BOE}$ coefficients here can be expressed in terms of correlators

\footnotetext{
${ }^{18}$ Note that this is the same redefinition as the previous subsection.
} 
of boundary operators in the original BCFT. ${ }^{19}$ Writing the cross ratio explicitly in terms of real coordinates, we have

$$
\eta=\frac{4 y_{1} y_{2}}{\left(x_{1}-x_{2}\right)^{2}+\left(y_{1}+y_{2}\right)^{2}}=1-\frac{\left(x_{1}-x_{2}\right)^{2}+\left(y_{1}-y_{2}\right)^{2}}{\left(x_{1}-x_{2}\right)^{2}+\left(y_{1}+y_{2}\right)^{2}},
$$

so we see that $\eta$ is a real number in $[0,1]$, with $\eta \rightarrow 1$ in the limit where $z_{1}$ and $z_{2}$ are much closer to each other than the boundary and $\eta \rightarrow 0$ in the limit where $z_{1}$ and $z_{2}$ are much closer to the boundary than to each other.

Consider the contribution from the term where only the boundary identity operator is kept in each BOE (2.14). This is equal to the disconnected term in the two-point function that factorizes into the product of one-point functions, and hence

$$
\begin{aligned}
\left\langle\Phi_{n}\left(z_{1}, \bar{z}_{1}\right) \bar{\Phi}_{n}\left(z_{2}, \bar{z}_{2}\right)\right\rangle_{\mathrm{UHP}, \mathbf{1}}^{b} & =\frac{\mathcal{B}_{\Phi \mathbf{1}}^{b} \mathcal{B}_{\Phi \mathbf{1}}^{b}}{\left[4 y_{1} y_{2}\right]^{d_{n}}} \\
& =\frac{g_{b}^{2(1-n)} \epsilon^{2 d_{n}}}{\left[4 y_{1} y_{2}\right]^{d_{n}}}
\end{aligned}
$$

where we have read off $\mathcal{B}_{\Phi \mathbf{1}}^{b}$ from (4.11). In general, this contribution should dominate the correlator in the limit $\eta \rightarrow 0$, where the two operators approach the boundary.

Bulk channel for the two-point function. We can obtain an alternative expression for the two-point function using the bulk OPE to express the product $\Phi_{n}\left(z_{1}, \bar{z}_{1}\right) \bar{\Phi}_{n}\left(z_{2}, \bar{z}_{2}\right)$ as a sum of bulk operators. This reduces the two-point function to a sum of one-point functions.

Using the general result (2.19) with (2.22) for this bulk-channel expression for the two-point function, we obtain

$$
\begin{aligned}
\left\langle\Phi_{n}\left(z_{1}, \bar{z}_{1}\right) \bar{\Phi}_{n}\left(z_{2}, \bar{z}_{2}\right)\right\rangle_{\mathrm{UHP}}^{b} & =\left[\frac{\eta}{4 y_{1} y_{2}}\right]^{d_{n}} \sum_{i} C_{\Phi_{n} \bar{\Phi}_{n}}^{i} \mathcal{A}_{i}^{b} \mathcal{F}\left(c, h_{i}, \frac{d_{n}}{2} \mid 1-\eta\right) \\
& =\left[\frac{1-\eta}{\left|z_{1}-z_{2}\right|^{2}}\right]^{d_{n}} \sum_{i} C_{\Phi_{n}}^{i} \bar{\Phi}_{n} \mathcal{A}_{i}^{b} \mathcal{F}\left(c, h_{i}, \frac{d_{n}}{2} \mid 1-\eta\right)
\end{aligned}
$$

where $i$ indexes untwisted operators in the $n$-fold product CFT. Again, it will be useful below to note the contribution where we keep only the bulk identity operator term in the OPE (2.16):

$$
\begin{aligned}
\left\langle\Phi_{n}\left(z_{1}, \bar{z}_{1}\right) \bar{\Phi}_{n}\left(z_{2}, \bar{z}_{2}\right)\right\rangle_{\mathrm{UHP}, \mathbf{1}}^{b} & =\frac{C_{\Phi_{n} \bar{\Phi}_{n}}^{\mathbf{1}} \mathcal{A}_{\mathbf{1}}^{b}}{\left|z_{1}-z_{2}\right|^{2 d_{n}}} \\
& =\frac{\epsilon^{2 d_{n}}}{\left|z_{1}-z_{2}\right|^{2 d_{n}}}
\end{aligned}
$$

where we have used $\mathcal{A}_{\mathbf{1}}^{b}=1$ and $C_{\Phi_{n} \bar{\Phi}_{n}}^{\mathbf{1}}=\epsilon^{2 d_{n}}$ from (4.9). This contribution should dominate the correlator in the limit $\eta \rightarrow 1$, where the two operators approach each other away from the boundary.

\footnotetext{
${ }^{19}$ To avoid cluttering our notation further, we will generally leave $n$ implicit in our BOE coefficients $\mathcal{B}$.
} 


\subsection{Rényi entropy}

We now use our results to calculate the Renyi entropy for an interval $A=\left[y_{1}, y_{2}\right]$ for the vacuum state of a BCFT on a half space $y>0$. This is related to the two-point function of twist operators on the upper half-plane as

$$
e^{(1-n) S_{A}^{(n)}}=\left\langle\Phi_{n}\left(z_{1}, \bar{z}_{1}\right) \bar{\Phi}_{n}\left(z_{2}, \bar{z}_{2}\right)\right\rangle_{\mathrm{UHP}}^{b},
$$

where we take $z_{1}=\left(0, y_{1}\right)$ and $z_{2}=\left(0, y_{2}\right)$.

Bulk and boundary limits. First, consider the Rényi entropy in the limits $\eta \rightarrow 0$ and $\eta \rightarrow 1$, where the twist operator two-point function is given by (4.16) and (4.18) respectively. In this case, we find that

$$
S_{A}^{(n)}= \begin{cases}\frac{c}{12} \frac{n+1}{n} \log \left(\frac{2 y_{1}}{\epsilon}\right)+\frac{c}{12} \frac{n+1}{n} \log \left(\frac{2 y_{2}}{\epsilon}\right)+2 \log g_{b} & \eta \rightarrow 0 \\ \frac{c}{6} \frac{n+1}{n} \log \left(\frac{\left|y_{2}-y_{1}\right|}{\epsilon}\right) & \eta \rightarrow 1 .\end{cases}
$$

Taking the $n \rightarrow 1$ limit, these give entanglement entropies

$$
S_{A}= \begin{cases}\frac{c}{6} \log \left(\frac{2 y_{1}}{\epsilon}\right)+\frac{c}{6} \log \left(\frac{2 y_{2}}{\epsilon}\right)+2 \log g_{b} & \eta \rightarrow 0 \\ \frac{c}{3} \log \left(\frac{\left|y_{2}-y_{1}\right|}{\epsilon}\right) & \eta \rightarrow 1 .\end{cases}
$$

We see that these precisely match the holographic results (3.8) and (3.9).

The result (3.8) is expected to be valid for any holographic CFT in some finite interval around $\eta=0$ where the RT surface is disconnected, while the result (3.9) is expected to be valid in a finite interval around $\eta=1$ in the case where the holographic theory can be modelled by a purely gravitational ETW brane. Thus, the results (4.21) have a much larger range of validity than we would naively expect from the CFT point of view. We would now like to understand from the CFT perspective how this larger range of validity for the vacuum results can arise.

Entropies at large $\boldsymbol{c}$. We begin with the general expressions (4.13) and (4.17) for the twist operator two-point function. General closed-form expressions for the conformal blocks are not known, but in the semiclassical limit $c \rightarrow \infty$, the chiral conformal blocks exponentiate [35]: ${ }^{20}$

$$
\mathcal{F}\left(c, h_{\mathrm{int}}, h \mid \eta\right) \stackrel{c \rightarrow \infty}{=} \exp \left[-\frac{c}{6} f\left(\frac{h_{\mathrm{int}}}{c}, \frac{h}{c}, \eta\right)\right] .
$$

The exponent $f$ is called the semiclassical block. ${ }^{21}$ In our case of identical external weights, recursion relations for the block allow one to commute the limits $c \rightarrow \infty$ and $h_{\text {int }} / c, h / c \rightarrow$

\footnotetext{
${ }^{20}$ There is a beautiful but non-rigorous argument for exponentiation from Liouville theory, using the explicit structure constants $[36,37]$ and the path integral. We refer the interested reader to the clear account in [38].

${ }^{21}$ In general, this depends on the set of external weights, but our notation takes into account that all of the external weights are identical.
} 
$0[10,39]$. Hence, the semiclassical blocks associated to light internal operators $h_{\text {int }}=O\left(c^{0}\right)$ are just the vacuum (semiclassical) block:

$$
f_{0}\left(\frac{h}{c}, \eta\right) \equiv f\left(0, \frac{h}{c}, \eta\right)
$$

We can apply these results to our two-point function of twist operators, for which all of the external dimensions are $d_{n} / 2$, and the central charge of the replicated CFT is $n c$.

We find that the $c \rightarrow \infty$ limit of the expressions (4.13) and (4.17) for the twist operator two-point function in the boundary and bulk channels become

$$
\begin{aligned}
& \left\langle\Phi_{n}\left(z_{1}, \bar{z}_{1}\right) \bar{\Phi}_{n}\left(z_{2}, \bar{z}_{2}\right)\right\rangle_{\mathrm{UHP}}^{b} \\
& =\left(\frac{\eta}{4 y_{1} y_{2}}\right)^{d_{n}}\left[\hat{\mathcal{D}}_{\mathrm{L}} e^{-\frac{n c}{6} f_{\hat{0}}\left(\frac{d_{n}}{2 n c}, \eta\right)}+\sum_{J_{\mathrm{H}}} \mathcal{B}_{\Phi J}^{b} \mathcal{B}_{\bar{\Phi} J}^{b} e^{-\frac{n c}{6} f\left(\frac{\hat{\Delta}_{J}}{n c}, \frac{d_{n}}{2 n c}, \eta\right)}\right] \\
& =\left(\frac{\eta}{4 y_{1} y_{2}}\right)^{d_{n}}\left[\mathcal{D}_{\mathrm{L}} e^{-\frac{n c}{6} f_{0}\left(\frac{d_{n}}{2 n c}, 1-\eta\right)}+\sum_{j_{\mathrm{H}}} C_{\Phi_{n}}^{j} \bar{\Phi}_{n} \mathcal{A}_{j}^{b} e^{-\frac{n c}{6} f\left(\frac{h_{j}}{n c}, \frac{d_{n}}{2 n c}, 1-\eta\right)}\right],
\end{aligned}
$$

where $J_{\mathrm{H}}, j_{\mathrm{H}}$ range over heavy internal operators, and $\hat{\mathcal{D}}_{\mathrm{L}}$ and $\mathcal{D}_{\mathrm{L}}$ are degeneracy factors multiplying the vacuum channel:

$$
\hat{\mathcal{D}}_{\mathrm{L}}=\sum_{J_{\mathrm{L}}} \mathcal{B}_{\Phi J}^{b} \mathcal{B}_{\bar{\Phi} J}^{b}, \quad \mathcal{D}_{\mathrm{L}}=\sum_{j_{\mathrm{L}}} C_{\Phi_{n} \bar{\Phi}_{n}}^{j} \mathcal{A}_{j}^{b} .
$$

As $c \rightarrow \infty$, the sums (4.24) and (4.25) should be dominated by the exponential with smallest exponent, if the coefficients of the exponential in the sum are not too large. More precisely, let us now make two assumptions:

1. The contribution of all heavy internal operators, in a neighbourhood around $\eta=0$ or $\eta=1$ in the respective channel, is exponentially suppressed in $c$. We will take heavy to mean any operators whose dimension scales as $O(c)$ or greater.

2. The degeneracy factors $\hat{\mathcal{D}}_{\mathrm{L}}, \mathcal{D}_{\mathrm{L}}$ are given by the vacuum contribution times some multiplicative correction that does not change the leading exponential in $c$ behaviour.

If the neighborhoods described in the first assumption meet at some point $\eta_{*}^{n}$, so that they cover the entire interval $\eta \in[0,1]$, we can conclude that large- $c$ behaviour of the correlator is given by the larger of the vacuum block contribution in the boundary channel or the vacuum block contribution in the bulk channel for the entire interval $\eta \in[0,1]$. This behaviour is commonly known as vacuum block dominance.

Under our first assumption of vacuum block dominance, the Rényi entropy for an interval $\left[y_{1}, y_{2}\right]$ is given by

$$
S_{A}^{(n)}=\left\{\begin{aligned}
& \frac{c}{6} \frac{n+1}{n} \log \left(y_{1}+y_{2}\right) \\
& \quad+\frac{c}{6} \frac{n}{n-1} f_{0}\left(\frac{1}{24}\left(1-\frac{1}{n^{2}}\right), \frac{4 y_{1} y_{2}}{\left(y_{1}+y_{2}\right)^{2}}\right)+\frac{1}{1-n} \log \hat{\mathcal{D}}_{\mathrm{L}} \quad \eta<\eta_{*}^{n} \\
& \frac{c}{6} \frac{n+1}{n} \log \left(y_{1}+y_{2}\right) \\
& \quad+\frac{c}{6} \frac{n}{n-1} f_{0}\left(\frac{1}{24}\left(1-\frac{1}{n^{2}}\right), \frac{\left(y_{1}-y_{2}\right)^{2}}{\left(y_{1}+y_{2}\right)^{2}}\right)+\frac{1}{1-n} \log \mathcal{D}_{\mathrm{L}}, \quad \eta>\eta_{*}^{n}
\end{aligned}\right.
$$


where $\eta_{*}^{n}$ is the value of $\eta$ at which the lower expression becomes larger than the upper one. In the limit $n \rightarrow 1$, the behavior of the semiclassical vacuum block follows from the result that for small $\alpha=(n-1) / 12$ [10],

$$
f_{0}(\alpha, \eta)=12 \alpha \log \eta+\mathcal{O}\left(\alpha^{2}\right),
$$

as we derive in section 5.2.

Under our second assumption of vacuum block dominance, we have that (at order $c$ )

$$
\begin{aligned}
\lim _{n \rightarrow 1} \frac{1}{1-n} \log \hat{\mathcal{D}}_{\mathrm{L}} & =\lim _{n \rightarrow 1} \frac{1}{1-n} \log \left(\mathcal{B}_{\Phi \mathbf{1}}^{b} \mathcal{B}_{\bar{\Phi} \mathbf{1}}^{b}\right) \\
& =-\frac{c}{3} \log \epsilon+2 \log g_{b} \\
\lim _{n \rightarrow 1} \frac{1}{1-n} \log \mathcal{D}_{\mathrm{L}} & =\lim _{n \rightarrow 1} \frac{1}{1-n} \log \left(C_{\Phi_{n}}^{\mathbf{1} \bar{\Phi}_{n}} \mathcal{A}_{\mathbf{1}}^{b}\right) \\
& =-\frac{c}{3} \log \epsilon
\end{aligned}
$$

up to contributions $O\left(c^{0}\right)$. Note that, by keeping the boundary entropy term, we are assuming that it, too, is $O(c)$.

Using these results and the results for the semiclassical blocks gives

$$
S_{A}=\lim _{n \rightarrow 0} S_{A}^{(n)}= \begin{cases}\frac{c}{6} \log \left(\frac{2 y_{1}}{\epsilon}\right)+\frac{c}{6} \log \left(\frac{2 y_{2}}{\epsilon}\right)+2 \log g_{b} & \eta<\eta_{*} \\ \frac{c}{3} \log \left(\frac{\left|y_{2}-y_{1}\right|}{\epsilon}\right) & \eta>\eta_{*}\end{cases}
$$

where $\eta_{*}$ is the value of $\eta$ where the two expressions coincide. These are exactly the results (4.21) we obtained keeping only the contributions from boundary and bulk identity operators. Thus, we see that the assumption of vacuum block dominance provides the extended range of validity for the formulas in (4.21), so that the results match our gravitational calculation with a purely gravitational ETW brane.

\subsection{BCFT requirements for vacuum block dominance}

Our expression in (4.29) now matches precisely the gravitational calculation, (3.8) and (3.9) for all $\eta$, at leading order in $c$. Following the previous work for bulk CFTs [10, 40], let us now explore what constraints our vacuum block dominance assumptions place on the spectrum and OPE data of the BCFT.

\subsubsection{Boundary channel}

We begin with the disconnected phase in the boundary channel that dominates in a neighbourhood of $\eta=0$. Our first assumption held that the contribution of heavy boundary operators was exponentially suppressed in $c$ and does not contribute at leading order. We will examine this claim in a cascading series of steps, from heaviest to lightest operators.

First, looking at operators of dimension $O\left(c^{\alpha}\right)$ for $\alpha>1$, we find that agreement with the gravity calculation seems to place rather weak constraints on the BCFT. In particular, 
the convergence of the boundary OPE can be used in an exactly analogous manner to the convergence of the bulk OPE [41] to show that the contribution of all operators of dimension $\hat{\Delta}>O(c)$ is exponentially suppressed in the central charge.

We then need only worry about operators up to dimension $O(c)$. Define $\rho_{b, n}(\delta) \mathrm{d} \delta$ to be the number of untwisted $n$-fold product boundary operators with dimensions $\hat{\Delta} \in$ $c[\delta, \delta+\mathrm{d} \delta]$, and define a measure of the average twist-operator BOE coefficients by

$$
\left|B_{n}(\delta)\right|^{2}=\frac{\sum_{\hat{\Delta}_{I} \in c[\delta, \delta+\mathrm{d} \delta]} \overline{\mathcal{B}}_{\Phi I}^{b} \overline{\mathcal{B}}_{\bar{\Phi} I}^{b}}{\sum_{\hat{\Delta}_{I} \in c[\delta, \delta+\mathrm{d} \delta]} 1} .
$$

where we have introduced $\overline{\mathcal{B}}_{\bar{\Phi} I}^{b}=\epsilon^{-d_{n}} g_{b}^{n-1} \mathcal{B}_{\bar{\Phi} I}^{b}$ to remove a universal prefactor that appears in all the BOE coefficients (see appendix B). We can use the known small $\eta$ expansion of the semiclassical block [10],

$$
f\left(h_{\mathrm{int}}, h_{\mathrm{ext}}, \eta\right)=6\left(2 h_{\mathrm{ext}}-h_{\mathrm{int}}\right) \log \eta-\frac{h_{\text {int }}}{2} \eta+\mathcal{O}\left(\eta^{2}\right),
$$

to write the bracketed expression in (4.24) as

$$
e^{-\frac{n c}{12}\left(1-\frac{1}{n^{2}}\right) \log \eta / \epsilon+2(1-n) \log g_{b}} \int_{0}^{O(1)} \mathrm{d} \delta \rho_{b, n}(\delta)\left|B_{n}(\delta)\right|^{2} e^{c \delta \log \eta+c \delta \eta+\mathcal{O}\left(\eta^{2}\right)} .
$$

In this expression, the heavy operators will not contribute to the order $c$ entanglement entropy if the integral over of any region bounded away from zero is exponentially suppressed in $c$ as compared to the integral near zero. This constrains the product of the density of operators appearing in the twist OPE and their OPE coefficients so as not to grow so quickly as to overcome the suppression from the block. For $\eta \ll 1$, this requires

$$
\log \left(\rho_{b, n}(\delta)\left|B_{n}(\delta)\right|^{2}\right)<c \delta \log \left(\eta^{-1}\right) \quad \text { for } \quad \delta \gtrsim 0 .
$$

In particular, requiring the CFT calculation to agree with the gravity result in an interval $0<\eta<\hat{\eta} \ll 1$ implies that $\rho_{b, n}(\delta)\left|B_{n}(\delta)\right|^{2}$ grows more slowly than $\exp (c \delta \log (1 / \hat{\eta})$. Extending to a larger range with $\hat{\eta}$ not necessarily much less than 1 gives a stronger constraint, but the exact form requires more detailed knowledge of the semiclassical block.

Let us then focus on the lower limit of this integral and consider only operators of dimension less than $O\left(c^{\alpha}\right)$ for $\alpha<1$, where we can approximate the semiclassical block by the vacuum block for all operators, up to $O\left(c^{\alpha-1}\right)$ corrections. The gravity calculation predicts that the leading exponential in $c$ behavior of the result matches the vacuum channel contribution, so we require that

$$
\sum_{I_{L}} \overline{\mathcal{B}}_{\Phi_{n} I}^{b} \overline{\mathcal{B}}_{\Phi_{n} I}^{b}
$$

is subexponential in $c .{ }^{22}$ In appendix B, we recall that the coefficients $\overline{\mathcal{B}}_{\Phi_{n} I}^{b}$ can be expressed in terms of $n$-point correlations functions of light boundary operators in the original BCFT,

\footnotetext{
${ }^{22}$ It is also interesting to consider the constraints on the CFT assuming that we have a conventional gravitational theory with a usual semiclassical expansion. In this case, the corrections to the entropy are expected to be of order $c^{0}$ (as opposed to some larger power of $c$ or $\log c$ ). In this case, we would obtain stronger constraints on the BCFT. However, for this paper, we focus on the constraints arising from demanding that the order $c$ terms in the entropies match with the classical gravity calculation.
} 
so this constraint can be translated into a constraint on the spectrum and $n$-point functions of the original BCFT. We consider the case $n=2$ in more detail below.

\subsubsection{Bulk channel}

We can largely repeat the above analysis in the bulk channel. Again, we find only weak constraints on operators of dimension $O\left(c^{\alpha}\right)$ for $\alpha>1$. The convergence of the bulk OPE can be used now precisely as in [41] to show that the contribution of all operators of dimension $\Delta>O(c)$ is exponentially suppressed in the central charge. We then need only worry about operators up to dimension $O(c)$.

Define $\rho_{n}(\delta) \mathrm{d} \delta$ to be the number of bulk untwisted $n$-fold product operators with dimensions $\Delta \in c[\delta, \delta+\mathrm{d} \delta]$ and

$$
A C_{b, n}(\delta)=\frac{\sum_{\Delta_{i} \in c[\delta, \delta+\mathrm{d} \delta]} \bar{C}_{\Phi \bar{\Phi}}^{i} \mathcal{A}_{i}^{b}}{\sum_{\Delta_{i} \in c[\delta, \delta+\mathrm{d} \delta]} 1}
$$

where $\bar{C}_{\Phi \bar{\Phi}}^{i}=\epsilon^{-2 d_{n}} C_{\Phi \bar{\Phi}}^{i}$. Then in this channel, we have

$$
e^{-\frac{n c}{12}\left(1-\frac{1}{n^{2}}\right) \log (1-\eta) / \epsilon} \int_{0}^{O(1)} \mathrm{d} \delta \rho_{n}(\delta) A C_{b, n}(\delta) e^{c \delta \log (1-\eta)+c \delta(1-\eta)+\mathcal{O}\left((1-\eta)^{2}\right)} .
$$

For heavy operators to not contribute to the order $c$ entanglement entropy when $1-\eta \ll 1$, we require

$$
\log \left(\rho_{n}(\delta) A C_{b, n}(\delta)\right)<c \delta \log (1-\eta)^{-1} \quad \text { for } \quad \delta \gtrsim 0 .
$$

This is analogous to the boundary channel condition, but with $\eta \rightarrow 1-\eta$.

For operators with dimension $O\left(c^{\alpha}\right)$ for $\alpha<1$, assumption 2 must hold in order to match with from gravity with a purely gravitational ETW brane. This requires that for the light operators, the sum

$$
\sum_{i_{L}} \bar{C}_{\Phi \bar{\Phi}}^{i} \mathcal{A}_{i}^{b}
$$

should be sub-exponential in $c$.

\subsection{Constraints on holographic BCFTs}

We have now spelled out explicitly a set of conditions on a BCFT that will ensure that the direct BCFT calculation of entanglement entropy matches with the gravity results in the holographic model with a purely gravitational ETW brane. However, we recall that the disconnected phase result (3.8) is universally valid for any holographic BCFT. Assuming that entanglement entropy has such a disconnected phase for some interval $\eta \in\left[0, \eta_{*}\right]$, as it does for the simple model, suggests that vacuum block dominance should hold for any holographic BCFT in an interval $\eta \in\left[0, \eta_{n}\right]$, where the upper end of the interval may depend on the Renyi index $n$.

From the results in the previous subsection, this implies a constraint

$$
\log \left(\rho_{b, n}(\delta)\left|B_{n}(\delta)\right|^{2}\right)<c \beta^{*} \delta+O\left(c^{a}\right), \quad a<1,
$$

where the quantities in the left side were defined in (4.30) and the preceeding paragraph. Here our knowledge of the semiclassical block was not sufficient to fix the $O(1)$ coefficient $\beta^{*}$ 
in this bound. In addition, we have a constraint (4.34) on the light operators. We take these bounds to be novel constraints on which BCFTs can possibly have a gravitational dual.

Although we found an analogous bound

$$
\log \left(\rho_{n}(\delta) A C_{b, n}(\delta)\right) \lesssim c \gamma^{*} \delta+O\left(c^{a}\right), \quad a<1
$$

in the bulk channel, this should not be viewed as a constraint on the boundary expectation values $\mathcal{A}_{i}^{b}$. While the disconnected phase is universal and depends only on the boundary entropy, the connected phase depends on the gravitational background (e.g. whether we have backreacting scalars in the solution dual to the BCFT vacuum). The vacuum solution for the bulk CFT is unique, but in contrast, there is no unique gravitational solution consistent with the symmetries of the BCFT.

A useful diagnostic for the non-universal behaviour of entropy and the bulk background is when light operators have large, $O(c)$, expectation values that backreact on the gravitational solution:

$$
\left\langle\mathcal{O}_{i}(x, y)\right\rangle_{\mathrm{UHP}}^{b}=\frac{\mathcal{A}_{i}^{b}}{(2 y)^{\Delta}}, \quad \mathcal{A}_{i}^{b} \sim c .
$$

Consistency with the large- $c$ factorization in the bulk then implies there is a large family of "multi-trace" operators of the schematic form $\mathcal{O}^{m}$ with expectation values $\left\langle\mathcal{O}^{m}\right\rangle_{\text {UHP }}^{b} \sim c^{m}$. When calculating the twist correlation function, this tower of operators must be resummed into a new semiclassical block, just as with the gravitational Virasoro descendants. For a BCFT, the form of the semiclassical block is theory-dependent and hence non-universal.

Thus, in the bulk channel vacuum-block dominance is not required by the theory. We must choose to restrict to those boundary states without semiclassical expectation values where non-universal contributions can be ignored. ${ }^{23}$

\subsubsection{Constraints on the BCFT base theory}

The constraint (4.39) involves both the spectrum of boundary operators in the $n$-copy theory and the BOE coefficients for twist operators in this theory. As we review in appendix $\mathrm{B}$, both of these can be related to the spectrum and OPE data for boundary operators in the single-copy BCFT; we can make use of these relations to convert the constraint (4.39) to a direct statement about the single-copy BCFT.

In particular, consider the case of $n=2$, where the branched geometry (including a regulator boundary for the twist operator as above) is conformal to the annulus. The Virasoro primaries appearing in the $n=2$ twist BOE, analogously to the bulk CFT case in $[40,42,43]$, contain products of base primaries of the form

$$
\mathcal{O}_{I}=\mathcal{O}_{i} \otimes \mathcal{O}_{i}
$$

For these operators, as we show in appendix B, the BOE coefficients are

$$
\overline{\mathcal{B}}_{\Phi_{2} I}^{b} \overline{\mathcal{B}}_{\Phi_{2} I}^{b}=16^{-2 \hat{\Delta}_{i}}
$$

\footnotetext{
${ }^{23}$ The same limitation holds for previous bulk CFT calculations. When light bulk operators have large expectation values that backreact on the geometry, the entanglement entropy of a region is no longer universal and is not determined by vacuum block dominance.
} 
identical to the bulk case in $[40,42,43]$ up to the non-standard normalization of the twist operators induced by the boundary. Taking into account only these primaries, we have a constraint from (4.34) that

$$
\sum_{i_{L}} 16^{-2 \hat{\Delta}_{i}}
$$

is sub-exponential in $c$, where the sum is over light boundary primary operators in the original BCFT. This will be true if the number of light boundary primaries in the base theory is also sub-exponential in $c$.

Note that the BOE also contains primaries composed of products of descendants in the base theory, such as

$$
\mathcal{O}_{i} \otimes L_{-1}^{2} \mathcal{O}_{i}-2 \frac{h_{i}+1 / 2}{h_{i}} L_{-1} \mathcal{O}_{i} \otimes L_{-1} \mathcal{O}_{i}+L_{-1}^{2} \mathcal{O}_{i} \otimes \mathcal{O}_{i}
$$

These are primaries with respect to the orbifold Virasoro generators:

$$
L_{m} \otimes 1+1 \otimes L_{m}
$$

but are generated by even powers of the antisymmetric linear combinations

$$
L_{m} \otimes 1-1 \otimes L_{m}
$$

To estimate the number of such primaries, we use the Hardy-Ramanujan Formula [44], which gives an asymptotic estimate for the number of descendants (partitions $p(k)$ ) at a given level $k$ :

$$
p(k) \sim e^{2 \pi \sqrt{k / 6}} .
$$

If the density of light primaries for a single BCFT is sub-exponential, as above, including the contribution of the extra primaries in the 2-copy BCFT not of the form (4.42) generates no new contributions exponential in $c$. Thus, we do not get a substantially stronger constraint from their inclusion.

\subsection{Black hole applications}

We have seen that under the assumption of vacuum block dominance, the direct BCFT calculation of entanglement entropy for an interval in the vacuum state of the theory on a half-line matches exactly with the gravity calculation using an effective bulk theory with a purely gravitational ETW brane.

In this section, we show that essentially the same BCFT calculation allows us to reproduce the gravitational results (3.11) and (3.12) for the entanglement entropy of an interval in a Euclidean time-evolved boundary state $\left|b, \tau_{0}\right\rangle$, and the results (3.14) and (3.15) for the entanglement entropy of the auxiliary radiation system in BCFT model of a twosided black hole coupled to a radiation bath.

From the CFT point of view, the three examples - half-space (section 3.2.1), cylinder (section 3.2.2), and thermofield double (section 3.2.3) - are essentially the same. The relevant path-integral geometries are shown in figure 8. In each case, the blue segment(s) show the region whose entanglement entropy we are computing. We insert twist operators 


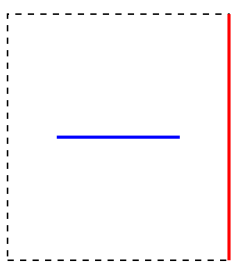

a

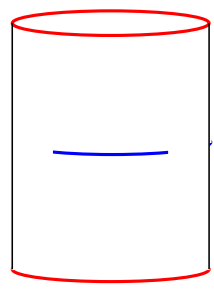

b

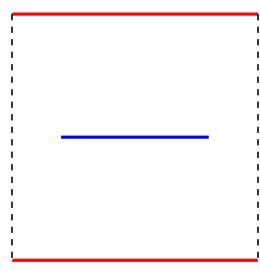

$\mathrm{c}$

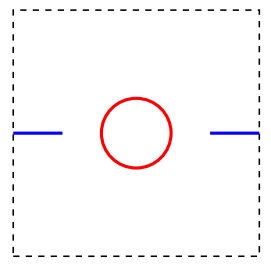

$\mathrm{d}$

Figure 8. Relation between Euclidean path integrals for the different BCFT setups in which we are calculating entanglement entropy. In (a), we have the half-space $x>0$. In (b), we have the path integral preparing the boundary state $\left|b, \tau_{0}\right\rangle$. We decompactify from a circle to a line to obtain the global quench geometry (c), which is equivalent (under a global conformal transformation) to (a). In $(\mathrm{d})$, we have the thermofield double state of (a).

$\Phi, \bar{\Phi}$ at the boundaries of these blue regions, and calculate entanglement entropy from their correlator.

In our first example, the entanglement entropy is calculated via the two-point function of twist operators on the half-plane $\{z=x+i y: \Im(z)=y>0\}$. The result for a holographic BCFT is

$$
\left\langle\bar{\Phi}_{n}\left(z_{1}\right) \Phi_{n}\left(z_{2}\right)\right\rangle_{\mathrm{UHP}}^{b}=\min \left\{\langle 0 \mid b\rangle^{2(n-1)}\left|\frac{4 y_{1} y_{2}}{\epsilon^{2}}\right|^{-d_{n}},\left|\frac{z_{12}}{\epsilon}\right|^{-2 d_{n}}\right\},
$$

for twist scaling dimension $d_{n}$. The calculation in section 3.2.3 reduces to exactly this correlator under the coordinate transformation

$$
w=f(z)=\frac{1}{z-i / 2}-i .
$$

This maps the boundary of the half-plane to the circle in figure 8d. Since twists are primary CFT operators (by definition), the correlator in the thermofield double geometry is related to the half-plane correlator by

$$
\left\langle\bar{\Phi}_{n}\left(w_{1}\right) \Phi_{n}\left(w_{2}\right)\right\rangle_{\mathrm{TFD}}^{b}=\left|w^{\prime}\left(z_{1}\right) w^{\prime}\left(z_{2}\right)\right|^{-d_{n}}\left\langle\bar{\Phi}_{n}\left(z_{1}\right) \Phi_{n}\left(z_{2}\right)\right\rangle_{\mathrm{UHP}}^{b} .
$$

For a symmetric interval with $w_{1,2}= \pm x_{0}+i t$, (4.49) and (4.51) agree with the holographic calculation in the $n \rightarrow 1$ limit [6].

The state $\left|b, \tau_{0}\right\rangle$ in section 3.2.2 takes the CFT on a circle. This is not related to the half-plane by a conformal transformation, so in principle the correlator of twists must be calculated anew. However, for a holographic CFT, the dual geometry for small $\tau_{0}$ is a trivial compacification of the covering space in which we "unwind" the angular coordinate. At leading order in large $c$, the entanglement entropy for an interval of fixed length on the circle is then independent of the circle's size. ${ }^{24}$

The limit of an infinite circle is the line. This gives the global quench geometry, as in figure $8 \mathrm{c}$. For small $\tau_{0}$, this is dual to a planar black hole. Unlike the cylinder geometry, the global quench is conformally equivalent to the half-plane, under the coordinate

\footnotetext{
${ }^{24}$ This is the phenomenon of large- $N$ volume independence. See, e.g., [45].
} 
transformation

$$
\kappa=\frac{2 \tau_{0}}{\pi} \log z
$$

The correlator of twists is then

$$
\left\langle\bar{\Phi}_{n}\left(\kappa_{1}\right) \Phi_{n}\left(\kappa_{2}\right)\right\rangle_{\mathrm{GQ}}^{b}=\left|\kappa^{\prime}\left(z_{1}\right) \kappa^{\prime}\left(z_{2}\right)\right|^{-d_{n}}\left\langle\bar{\Phi}_{n}\left(z_{1}\right) \Phi_{n}\left(z_{2}\right)\right\rangle_{\mathrm{UHP}}^{b} .
$$

Once again, combining this with (4.49) gives the same result as the HRT formula [5].

\section{Multiple intervals}

The generalization of our BCFT results from a single interval to multiple intervals closely parallels the generalization of the CFT result from two intervals to multiple intervals [10]. We start with the holographic calculation, and then discuss how to obtain the results from the monodromy method in the BCFT.

\subsection{Holographic results for multiple intervals}

Consider a collection of $k$ disjoint intervals $A=\sqcup_{i} A_{i}, A_{i}=\left[x_{2 i-1}, x_{2 i}\right]$, in the vacuum state of a BCFT on the half-space $x \geq 0$, with an associated minimal surface $\mathcal{X}_{A}$. A given topology for $\mathcal{X}_{A}$ geodesically (and without intersection) pairs each endpoint $x_{i}$ to either (a) another endpoint $x_{j}$, or (b) the brane. Morally, we can view the latter as pairing $x_{i}$ to an image point $x_{i}^{*}$ placed on a mirror image of the bulk theory across the brane.

Thus, the possible topologies $\operatorname{top}\left(\mathcal{X}_{A}\right)$ can equally be described by symmetric geodesic pairings of $2 k$ intervals, of which there are $\left(\begin{array}{c}2 k \\ k\end{array}\right) .{ }^{25}$ Assuming that the gravity dual theory is described via a purely gravitational ETW brane, so that the local geometry is pure AdS, the two types of geodesics have (regulated) lengths

$$
\frac{\ell_{i j}}{4 G_{\mathrm{N}}}=\frac{c}{3} \log \left(\frac{x_{i j}}{\epsilon}\right), \quad \frac{\ell_{m m^{*}}}{4 G_{\mathrm{N}}}=\frac{c}{6} \log \left(\frac{2 x_{m}}{\epsilon}\right)+\log g_{b} .
$$

Hence, the holographic result is

$$
\begin{aligned}
S_{A} & =\min _{\operatorname{top}\left(\mathcal{X}_{A}\right)} \frac{1}{4 G_{\mathrm{N}}}\left[\sum_{(i j)} \ell_{i j}+\sum_{\left(m m^{*}\right)} \ell_{m m^{*}}\right] \\
& =\min _{\operatorname{top}\left(\mathcal{X}_{A}\right)}\left[\sum_{(i j)} \frac{c}{3} \log \left(\frac{x_{i j}}{\epsilon}\right)+\sum_{\left(m m^{*}\right)} \frac{c}{6} \log \left(\frac{2 x_{k}}{\epsilon}\right)+\log g_{b}\right],
\end{aligned}
$$

where $(i j)$ denotes paired endpoints in the half-space and $\left(m m^{*}\right)$ image-paired endpoints.

As a concrete example, take the interval $A=\left[x_{1}, x_{2}\right]$. The explicit expression for holographic entanglement entropy is then

$$
\begin{aligned}
S_{A} & =\min \left\{\frac{c}{3} \log \left(\frac{x_{2}-x_{1}}{\epsilon}\right), \frac{c}{6} \log \left(\frac{4 x_{1} x_{2}}{\epsilon^{2}}\right)+2 \log g^{b}\right\} \\
& =\min \left\{S_{A}^{\text {conn }}, S_{A}^{\text {disc }}\right\},
\end{aligned}
$$

\footnotetext{
${ }^{25}$ Arbitrary non-intersecting geodesic pairings of $n$ intervals are counted by Catalan numbers $\left(\begin{array}{c}2 k \\ 2\end{array}\right) /(k+1)$. Each yields $k+1$ symmetric pairings on $2 k$ intervals, giving our result. We thank Chris Waddell for discussion of this point.
} 
recovering our results from section 3.2.1. The calculation is similar in other vacuum geometries. We can also include a boundary-centred interval $\left[0, x_{0}\right]$, which forces at least one image-paired geodesic. ${ }^{26}$

\subsection{BCFT calculation for multiple intervals}

To calculate the entanglement entropy of $A=\sqcup A_{i}$ in the BCFT on a half-space, we can simply calculate a correlator of $k$ twist and anti-twist operators on the Euclidean UHP and analytically continue. We will therefore focus on the UHP calculation. As above, we can use kinematic doubling to write the correlator as

$$
\left\langle\prod_{i=1}^{k} \Phi_{n}\left(z_{2 i-1}, \bar{z}_{2 i-1}\right) \bar{\Phi}_{n}\left(z_{2 i}, \bar{z}_{2 i}\right)\right\rangle_{\mathrm{UHP}}^{b}=\left\langle\prod_{i=1}^{k} \Phi_{n}\left(z_{2 i-1}\right) \bar{\Phi}_{n}\left(\bar{z}_{2 i-1}\right) \bar{\Phi}_{n}\left(z_{2 i}\right) \Phi_{n}\left(\bar{z}_{2 i}\right)\right\rangle .
$$

As in the single interval case, we have some choice about the order in which we perform bulk OPE or BOE expansions of the twist correlator. We can regard this sequence of choices as a fusion channel $\mathcal{E}$, analogous to the s- and t-channels in the single interval case. A given fusion channel has a natural expansion in terms of a set of cross-ratios, $\vec{\eta}$, and higher-point conformal blocks:

$$
\left\langle\prod_{i=1}^{k} \Phi_{n}\left(z_{2 i-1}\right) \bar{\Phi}_{n}\left(\bar{z}_{2 i-1}\right) \bar{\Phi}_{n}\left(z_{2 i}\right) \Phi_{n}\left(\bar{z}_{2 i}\right)\right\rangle_{\mathrm{UHP}}^{b}=N(\vec{\eta}) \sum_{\vec{h}, \vec{\Delta}} \mathcal{C}^{\mathcal{E}, \vec{h}, \vec{\Delta}} e^{-\frac{n c}{6} f\left(\vec{h}, d_{n} / 2 n c, \vec{\eta}\right)},
$$

where we have taken the semiclassical limit, and $\mathcal{C}^{\mathcal{E}, \vec{h}, \vec{\Delta}}$ is a product of OPE and BOE coefficients depending on the internal weights $\vec{h}, \vec{\Delta}$. Here $N(\vec{\eta})$ is just a standard prefactor.

Having related the UHP correlator to a chiral correlator, the higher-point blocks can be obtained from the standard monodromy method. We briefly summarize this method here, following [10]. (We discuss the method in slightly more detail in appendix C.) Readers familiar with the monodromy method may freely jump ahead to (5.10).

The monodromy method begins with a powerful trick: instead of the desired $2 k$-point function, consider a $(2 k+1)$-point function, where we have added an additional operator, $\chi_{(1,2)}(z)$, which is taken to be a null descendant of a primary operator $\theta(z) .{ }^{27}$ The null operator must decouple and the correlator must vanish. The vanishing of the correlator is expressed as the differential equation (writing $\chi(z)$ as a differential operator acting on $\theta(z)$ )

$$
\Theta^{\prime \prime}(z)+T(z) \Theta(z)=0
$$

where $\Theta(z)$ is the correlator

$$
\Theta(z)=\left\langle\theta(z) \prod_{i} \Phi_{n}\left(z_{2 i-1}\right) \bar{\Phi}_{n}\left(\bar{z}_{2 i-1}\right) \bar{\Phi}_{n}\left(z_{2 i}\right) \Phi_{n}\left(\bar{z}_{2 i}\right)\right\rangle,
$$

\footnotetext{
${ }^{26}$ When $A=\left[0, x_{0}\right] \sqcup A_{1} \sqcup \cdots \sqcup A_{k-1}, \mathcal{X}_{A}$ has $\left(\begin{array}{c}2 k+1 \\ k\end{array}\right)$ possible topologies. This can established by similar combinatorics to the non-boundary case.

${ }^{27}$ Strictly speaking, this operator is only guaranteed to exist in Liouville theory. However, as the block is a kinematic object, we expect the form not to depend on whether this operator exists in our theory or not.
} 
and $T(z)$ is

$$
T(z)=\sum_{i}\left\{\frac{6 h_{n} / c}{\left(z-z_{i}\right)^{2}}+\frac{6 h_{n} / c}{\left(z-\bar{z}_{i}\right)^{2}}+\frac{\partial_{z_{i}}}{z-z_{i}}+\frac{\partial_{\bar{z}_{i}}}{z-\bar{z}_{i}}\right\}
$$

For a given channel $\mathcal{E}$, in an appropriate limit of the cross ratios $\eta \rightarrow \eta_{0}^{\mathcal{E}}$, we expect this to be dominated by the exchange of the lightest possible operator, generally the identity and its descendants. We thus make the ansatz that the correlator is given by

$$
\Theta(z) \approx \psi\left(z \mid z_{i}, \bar{z}_{i}\right) e^{-\frac{n c}{3} f_{0}^{\mathcal{E}}}
$$

to leading order in $c$. Here $f_{0}^{\mathcal{E}}$ is the semiclassical vacuum block for the original $2 k$-point function and $\psi\left(z \mid z_{i}, \bar{z}_{i}\right)$ is thought of as a 'wavefunction' for the inserted operator. In this case, we can rewrite $T(z)$

$$
T(z)=\sum_{i}\left\{\frac{6 h_{n} / c}{\left(z-z_{i}\right)^{2}}+\frac{6 h_{n} / c}{\left(z-\bar{z}_{i}\right)^{2}}-\frac{c_{i}}{z-z_{i}}-\frac{\bar{c}_{i}}{z-\bar{z}_{i}}\right\}
$$

where the $c_{i}$ are accessory parameters:

$$
c_{i}=\frac{\partial f_{0}^{\mathcal{E}}}{\partial z_{i}}, \quad \bar{c}_{i}=\frac{\partial f_{0}^{\mathcal{E}}}{\partial \bar{z}_{i}}=\overline{c_{i}} .
$$

If we know the accessory parameters, we can integrate (5.9) to find the block $f_{0}^{\mathcal{E}}$. To determine these parameters, the monodromy method then uses the fact that a solution of the differential equation must have monodromies around any set of points that is consistent with the corresponding operator being exchanged in the block. This constraint can be used to fix the accessory parameters. In general, this cannot be done analytically. However, it is possible to find the parameters explicitly for twist operators in the $n \rightarrow 1$ limit, when we can break the problem down into a sum of independent monodromy constraints.

Solving the monodromy constraints and integrating the accessory parameters near $n=1$, one finds

$$
f_{0}^{\mathcal{E}}=\sum_{(i j)} 12 \alpha \log \left|z_{i j}\right|^{2}+\sum_{\left(m m^{*}\right)} 12 \alpha \log z_{m m^{*}}+O\left(\alpha^{2}\right) .
$$

Here, the channel $\mathcal{E}$ pairs some twists to anti-twists on the same half-plane, and some twists to their images on the opposite half-plane. We have denoted the pairs by $(i j)$ and $\left(\mathrm{mm}^{*}\right)$ respectively, and note that (as expected from the CFT case [10]) channels biject with the topologies of section 5.1, so we can view $\mathcal{E} \in \operatorname{top}\left(\mathcal{X}_{A}\right)$. We illustrate the correspondence between channels, trivial cycles, and the bulk RT surfaces in figure 9 .

To calculate the entanglement entropy, we also need to compute $\mathcal{C}_{0}^{\mathcal{E}}$. This is easily done, since the OPE coefficients for vacuum exchange are always unity, while the BOE always gives the one-point function of twists (4.11). If there are $M$ image pairs $\left(\mathrm{mm}^{*}\right)$, we have

$$
\mathcal{C}_{0}^{\mathcal{E}}=\left[g_{b}^{1-n}\right]^{M}=g_{b}^{-12 \alpha M}
$$



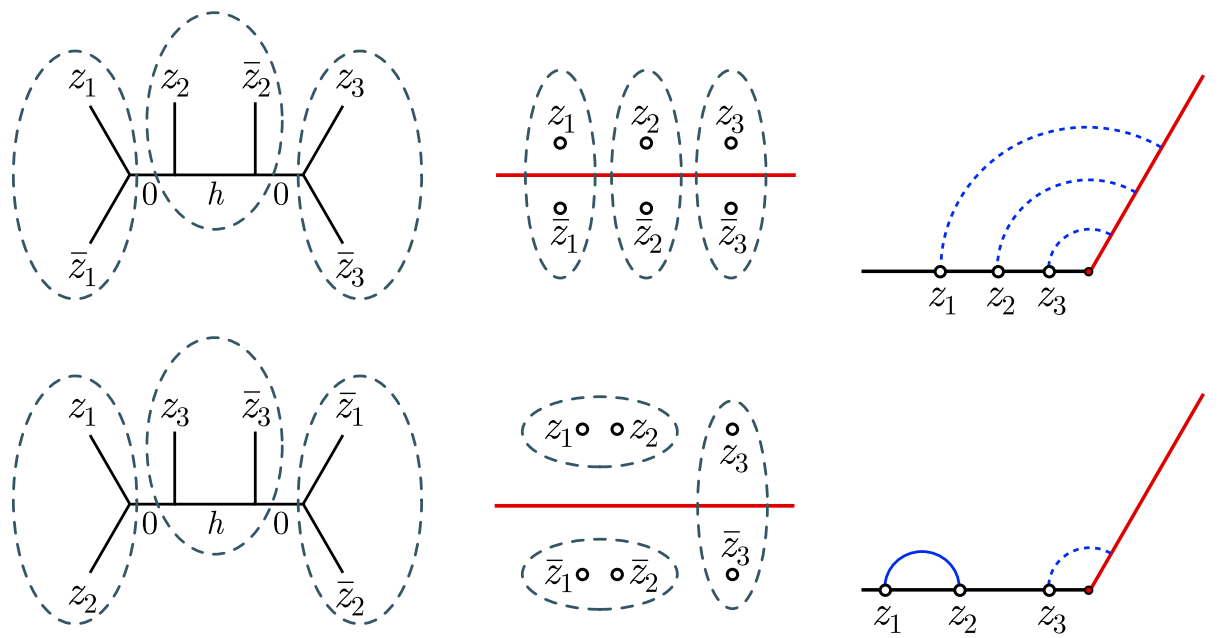

Figure 9. Left. Vacuum exchange in two different channels for $k=3$ twists on the UHP. Trivial cycles cut through identities. Middle. The monodromy cycles to be trivialized in the doubled picture of the BCFT. Right. The corresponding RT topologies in the bulk with an ETW brane.

We can recover the factors of $\epsilon$ from the one-point functions (4.8) and (4.11). From (5.7), the entanglement entropy in the limit $\eta \rightarrow \eta_{0}^{\mathcal{E}}$ is then

$$
\begin{aligned}
S_{A} & =\lim _{\alpha \rightarrow 0}\left(\frac{c}{36 \alpha} f_{0}^{\mathcal{E}}-\frac{1}{12 \alpha} \log \mathcal{C}_{0}^{\mathcal{E}}\right) \\
& =\sum_{(i j)} \frac{c}{3} \log \left(\frac{\left|z_{i j}\right|}{\epsilon}\right)+\sum_{\left(m m^{*}\right)} \frac{c}{6} \log \left(\frac{z_{m m^{*}}}{\epsilon}\right)+\log g_{b} .
\end{aligned}
$$

The corrections to (C.4) are in $\alpha^{2}$ and not in $z_{i j}$. It follows that in finite regions around $\eta_{0}^{\mathcal{E}}$, expression (5.12) is the full entanglement entropy to leading order in $c$.

If we make the assumption of vacuum block dominance as in section 4.3 , we can upgrade (5.12) to precisely reproduce (5.1):

$$
S_{A}=\min _{\operatorname{top}\left(\mathcal{X}_{A}\right)}\left[\sum_{(i j)} \frac{c}{3} \log \left(\frac{\left|z_{i j}\right|}{\epsilon}\right)+\sum_{\left(m m^{*}\right)} \frac{c}{6} \log \left(\frac{z_{m m^{*}}}{\epsilon}\right)+\log g_{b}\right] .
$$

This follows because vacuum dominance in a channel $\mathcal{E}$ implies the vacuum contribution is larger in other channels. Thus, we have a derivation of the full RT formula in a BCFT dual to AdS with an ETW brane, to the same level of generality as the CFT case [10].

\section{Replica calculation in the gravity picture}

In this paper, we have seen how phase transitions in holographic BCFT entanglement entropies, originally understood using the HRT formula, can be understood directly in the BCFT via the exchange of dominance between bulk and boundary channels in the two-point function of twist operators. The twist operator correlation function calculates the Rényi entropies as the partition function for the BCFT on a replica manifold. Our 
calculation indicates that the Rényi entropies themselves, or the replica partition functions, also have a phase transition. ${ }^{28}$ It is interesting to understand the gravitational origin of these transitions. Here, we use the fact that the CFT partition function on the replica manifold should be equal to the partition function of the gravity theory in which the spacetime geoemtries are constrained to be asymptotically AdS, with boundary geometry equal to the replica manifold.

In [6], inspired by $[12,13]$, it was argued that the transitions in the gravitational path integral arise because we can have various topologies for the ETW brane whose boundary is the disconnected set of $n$ CFT boundaries, where $n$ is the replica index. In the saddle-point approximation (which gives the leading contribution to the partition function in the $1 / c$ expansion), the logarithm of the CFT partition function is equal to the gravitational action for the bulk configuration with least action. The transition occurs when the gravitation configuration with least action changes as the parameters specifying the interval are varied.

In this section, we will confirm these expectations in an explicit example where the geometries can be understood in detail. We consider the Rényi entropy for a single interval $\left[x_{1}, x_{2}\right]$ in the vacuum state of a BCFT on a half-line $x>0$. We assume that the BCFT and the chosen boundary condition correspond to a dual gravitational theory that has an effective three-dimensional description as gravity with a negative cosmological constant and an gravitational ETW brane with zero tension. In this simple case, the dual geometries that we need can be obtained very simply from the geometries that contribute to the calculation of the Rényi entropies for a pair of intervals $\left[-x_{2},-x_{1}\right] \cup\left[x_{1}, x_{2}\right]$ in the vacuum state of our parent $\mathrm{CFT}$ on a real line. These geometries have a $\mathbb{Z}_{2}$ symmetry corresponding to the transformation $x \rightarrow-x$ in the CFT that exchanges the intervals.

It follows from symmetry that the bulk surface fixed by this transformation has zero extrinsic curvature. ${ }^{29}$ Thus, if we consider a new geometry defined as half of the previous geometry, with a zero-tension ETW brane replacing the $\mathbb{Z}_{2}$ symmetric surface, the new geometry will satisfy the gravitational equations in the bulk and at the ETW brane. The new geometry is thus a valid saddle-point geometry for the BCFT Rényi entropy calculation. It is plausible that all of the geometries we need can be obtained in this way.

Happily, the geometries for the two-interval calculation have already been discussed in detail by Faulkner in [11]. There, it was understood that the phase transition in Rényi entropies indeed arises from a transition in the lowest-action gravitational solution. We can check that under this transition, the hypersurface fixed by the $\mathbb{Z}_{2}$ symmetry changes topology, becoming connected when the intervals are close to each other. We illustrate this for the case of the second Renyi entropy in figure 10. Thus, the ETW brane in the gravity version of the BCFT calculation takes on a replica wormhole geometry in the gravitational saddle that computes the entanglement entropy of an interval close to the boundary.

There is an alternative intuitive picture for the transition in the ETW-brane topology that leads to the phase transition in Rényi entropies. Consider again the two-interval

\footnotetext{
${ }^{28}$ Note that the parameter values at which this happens depends on the replica index.

${ }^{29}$ To see this, recall that the extrinsic curvature can be defined as the Lie derivative of the hypersurface metric with respect to a normal vector $n$ to the hypersurface. This switches sign under $n \rightarrow-n$, but the extrinsic curvature is invariant under this operation because of the symmetry.
} 


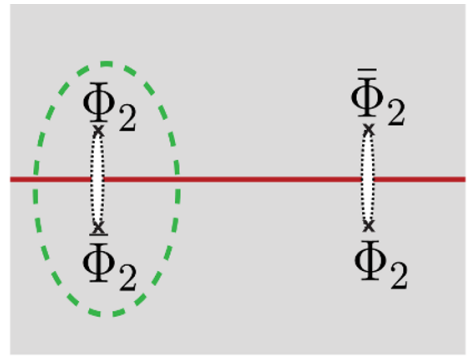

(a)

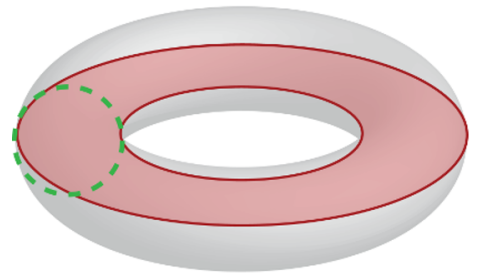

(c)

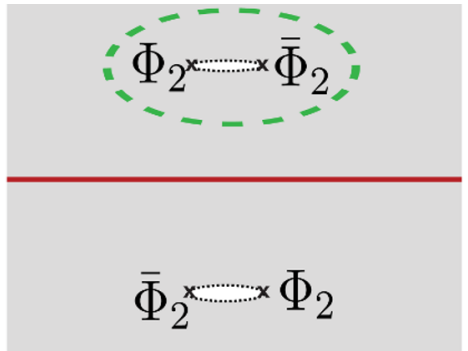

(b)

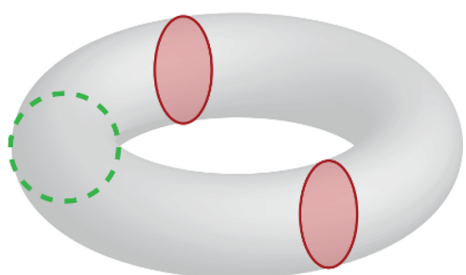

(d)

Figure 10. CFT and bulk depictions of the path integral for computing the second Rényi entropy of two intervals. We indicate a $\mathbb{Z}_{2}$ identification, whose fixed-point locus is drawn as a red line, that relates this to a BCFT whose boundary is the red line. (a) When the twist operators are near the $\mathbb{Z}_{2}$-line (in red), the corresponding bulk solution will fill in the cycle indicated by the green dashed line. The $\mathbb{Z}_{2}$-line and the bulk contractible cycle intersect each other. (b) When the twist operators are far from the $\mathbb{Z}_{2}$-line, the bulk contractible cycle (green) is now homologous to the $\mathbb{Z}_{2}$-line on each sheet. (c) The corresponding bulk solution when the twist operators are near the $\mathbb{Z}_{2}$-line. The bulk $\mathbb{Z}_{2}$-surface connects the two $\mathbb{Z}_{2}$-lines on each sheet. (d) The bulk solution when the twist operators are far from the $\mathbb{Z}_{2}$-line. There is now a disconnected bulk $\mathbb{Z}_{2}$-surface for each $\mathbb{Z}_{2}$-line on the boundary.

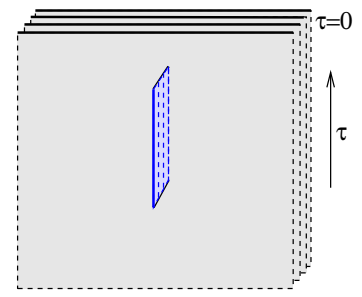

Figure 11. Entangled state of $n$ CFTs produced by inserting twist and anti-twist operators into the Euclidean path integral for the vacuum state. As the branch cut is moved toward $\tau=0$, the entanglement between the CFTs becomes large, and the $\tau=0$ spatial slice of the dual geometry becomes a connected multi-boundary wormhole. In the original picture, this surface is the ETW brane geometry in the gravitational calculation of Rényi entropy for an interval close to the BCFT boundary. 
calculation, but now reinterpret the $x$ coordinate as a Euclidean time coordinate $\tau$. In this case, the CFT path-integral on the $\tau<0$ part of the replica manifold can be interpreted as a Euclidean path integral that creates a state of $n$ CFTs. This is illustrated in figure 11. The $\mathbb{Z}_{2}$ symmetric surface in the dual gravitational geometry is the $\tau=0$ surface that gives the initial data for the time-symmetric Lorentzian geometry dual to this state.

In the limit where our interval moves toward $\tau=-\infty$, the path integral will give the vacuum state of $n$ CFTs, so the dual geometry is $n$ copies of AdS and the $\mathbb{Z}_{2}$ symmetric surface has $n$ disconnected components. On the other hand, as the interval moves closer to $\tau=0$, the path integral produces a state with more and more entanglement between the $n$ CFTs ${ }^{30,31}$ At some point, we have a phase transition similar to the Hawking-Page transition, where the $\tau=0$ spatial slice in the dual geometry becomes connected and the Lorentzian geometry dual to our state is a multi-boundary wormhole. In our BCFT application, this $\tau=0$ spatial slice becomes the ETW brane geometry, so we see that the Euclidean wormholes in the replica calculation can be directly related to the usual appearance of wormholes in the gravity dual of highly-entangled states of holographic systems.

\section{Discussion}

Starting with the vacuum state of a 1+1-dimensional CFT, the geometry of a putative bulk dual is fixed by symmetry to be $\mathrm{AdS}_{3}$, up to internal dimensions. We can also fix $\mathrm{AdS}_{3}$ using the RT formula: it is the unique bulk geometry whose minimal surfaces correctly reproduce the universal result for the entanglement entropy of a single interval. The RT formula makes non-universal predictions for two or more intervals, so we can go in the other direction and determine the class of holographic CFTs which reproduce these non-universal gravitational results. As shown in [10], vacuum block dominance guarantees that the twist-antitwist correlators used to calculate entanglement entropy agree with the holographic value for any number of intervals. Vacuum dominance places explicit constraints on the spectrum and OPE coefficients of a holographic CFT.

The logic for a CFT with boundary, and a conformally invariant boundary state, is similar. Symmetry, or the universal result for the entanglement entropy of a boundarycentred interval, restricts us to a class of $\mathrm{SO}(1,2)$-invariant geometries. These can include warping in the bulk and compact internal dimensions as before. The simplest bulk geometry is a portion of $\mathrm{AdS}_{3}$ cut off by an ETW brane with purely gravitational couplings [3], though we emphasize this is not the most general bulk dual consistent with ground-state symmetry.

In this paper, we have taken the next step of transforming non-universal gravitational predictions from these boundary-state geometries (with a purely gravitational ETW brane) into a constraint on holographic BCFTs. To match the holographic predictions for a noncentred interval, or indeed any number of intervals, vacuum dominance in both the BCFT

\footnotetext{
${ }^{30}$ For example, in the $n=2$ case, we get a path integral similar to the one that produces the thermofield double state, and moving the interval closer to $\tau=0$ corresponds to increasing the temperature.

${ }^{31}$ In the limit where the interval hits $\tau=0$, we have a state where the left half of $\mathrm{CFT}_{k}$ is connected to the right half of $\mathrm{CFT}_{k+1}$, so the entanglement of the system formed from the left and right half of $\mathrm{CFT}_{k}$ is infinite.
} 
bulk and boundary channels is necessary and sufficient. From a kinematic perspective, this follows from the doubling trick and the remaining copy of the Virasoro algebra. But the implications for the BCFT spectrum and OPE coefficients are more subtle. We have made some precise statements above, but expect there is more juice to be squeezed from this particular lemon. For instance, it might be possible to finesse the spectral constraints along the lines of [46], though the CFT machinery required is potentially quite different. It would also be interesting to investigate the additional constraints that arise from assuming not only that the BCFT calculations reproduce the leading $O(c)$ entropies, but also that the subleading corrections to the entropies are order $c^{0}$ as we expect from a conventional gravitational theory with a semiclassical expansion.

We have argued that the expression (3.8) for the small $\eta$ entanglement entropy is universal in holographic CFTs (assuming that the RT surface is disconnected in some interval $\left[0, \eta_{d}\right]$ as in the simple model), so the constraints associated with vacuum block dominance for an interval around $\eta=0$ should be expected to hold much more generally, for any holographic BCFT with a disconnected RT surface phase at small $\eta$. It seems plausible that any holographic BCFT should have such a phase, though it would be interesting to find a direct argument.

Our results have several interesting consequences and applications. First, they put the AdS/BCFT proposal of [3] on firmer microscopic footing, exhibiting explicit conditions on a BCFT under which a locally AdS geometry with a purely gravitational ETW brane captures the microscopic ground-state entanglement entropies. The gravity calculations allow the RT surface to end on an ETW brane, so our results also confirm this aspect of Takayanagi's proposal. ${ }^{32}$

Our work also has direct applications to the physics of black holes. For black holes dual to CFT states prepared by a Euclidean path integral on the cylinder with conformally invariant boundary conditions, the phase transition in entanglement entropy for a noncentred interval leads to a period of Lorentzian time where boundary observers with access to suitably large boundary regions can see behind the horizon [5].

Similarly, for the thermofield-double state of a BCFT on a half-line, the phase transition in entanglement entropy corresponds to a transition in bulk entanglement wedge to include part of the black hole interior [6]. Treating this as a model of a black hole in equilibrium with its Hawking radiation, after this transition part of the interior is reconstructable from the radiation. The brane is therefore playing a similar role to the "quantum extremal islands" which restore unitarity of the Page curve [7-9]. We have argued above that the phase transition in entanglement entropy can be directly related to topological changes in bulk replica wormholes, and it is of obvious interest to explore this connection further.

\section{Acknowledgments}

We would like to thank Tarek Anous, Thomas Hartman, Eliot Hijano, Alex May, Dominik Neuenfeld, and Chris Waddell for useful discussions. DW is supported by an International

\footnotetext{
${ }^{32}$ From the lower-dimensional perspective, this is a modification of the usual homology condition, though no modification is required if we take the higher-dimensional perspective that the ETW brane represents a smooth part of the full bulk geometry.
} 
Doctoral Fellowship from the University of British Columbia. MVR is supported by the Simons Foundation via the It From Qubit Collaboration and a Simons Investigator Award. This work is supported in part by the Natural Sciences and Engineering Research Council of Canada.

\section{A BCFT two-point functions from Virasoro conformal blocks}

In this appendix, we briefly review the structure of four-point functions of chiral operators and their expansion in terms of Virasoro conformal blocks, and then argue that the same objects form the building blocks of two-point functions in boundary conformal field theories.

Chiral four-point functions and conformal blocks. In a 2D CFT, for operators $\phi_{i}$ with chiral dimensions $h_{i}$, the global conformal symmetry implies that the four-point function takes the form

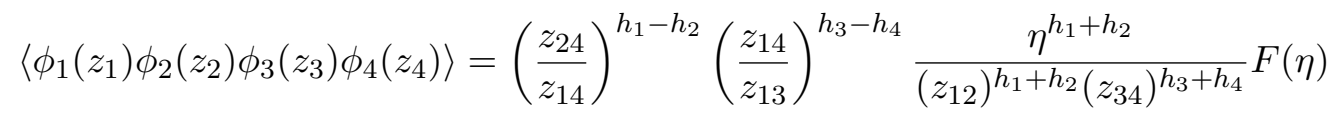

where $z_{i j}=z_{i}-z_{j}$ and $F$ is some function of the single cross-ratio $\eta=z_{12} z_{34} /\left(z_{13} z_{24}\right)$. We can define $F$ as

$$
F(\eta)=\lim _{z_{\infty} \rightarrow \infty}(-1)^{h_{1}+h_{2}+h_{3}+h_{4}} z_{\infty}^{2 h_{4}}\left\langle\phi_{1}(0) \phi_{2}(\eta) \phi_{3}(1) \phi_{4}\left(z_{\infty}\right)\right\rangle .
$$

We can express $\mathcal{F}$ in terms of the OPE data for the CFT and a standard set of functions by expanding the products $\phi_{1}\left(z_{1}\right) \phi_{2}\left(z_{2}\right)$ and $\phi_{3}\left(z_{3}\right) \phi_{4}\left(z_{4}\right)$ using (2.16). In this case, the four point function reduces to a sum of two-point functions of intermediate operators,

$$
F(\eta)=\sum_{i} C_{12}^{i} C_{34}^{i} \mathcal{F}\left(c, h ;\left[h_{1}, h_{2}, h_{3}, h_{4}\right] \mid \eta\right)
$$

The conformal blocks $\mathcal{F}\left(c, h ;\left[h_{1}, h_{2}, h_{3}, h_{4}\right] \mid \eta\right)$ are specific functions which depend only on the central charge, the dimensions $h_{i}$ of the external operators, and the "internal" dimension $h$. These give the contribution to the four-point function from a primary operator of weight $h$ and all of its Virasoro descendants. The block has a simple behavior in the limit $\eta \rightarrow 0$, where we have

$$
\mathcal{F}\left(c, h ;\left[h_{1}, h_{2}, h_{3}, h_{4}\right] \mid \eta \rightarrow 0\right) \sim \eta^{h-h_{1}-h_{2}}
$$

BCFT two-point function. We now consider the two-point function of bulk operators in a BCFT defined on the upper-half-plane

$$
\left\langle\mathcal{O}_{1}\left(z_{1}, \bar{z}_{1}\right) \mathcal{O}_{2}\left(z_{2}, \bar{z}_{2}\right)\right\rangle_{\mathrm{UHP}}^{b}
$$

As we discussed in section 2, this has the kinematics of a chiral four-point function. We will show this somewhat more carefully here, and see that we can expand the two-point function in either a bulk channel or a boundary channel in terms of the chiral conformal blocks defined above. 
One-point functions for scalar Virasoro descendants. To begin, it will be useful to compute one-point functions for scalar global primaries that are themselves Virasoro descendants. Consider, in particular, a Virasoro primary operator $\mathcal{O}_{h, \bar{h}}(z, \bar{z})$ and state $|h, \bar{h}\rangle$ for the CFT on $S^{1}$ associated to it by the state-operator correspondence. We denote an operator $\mathcal{O}_{h, \bar{h}}^{\alpha, \beta}(0)$ which creates the Virasoro descendants of this state $V_{\alpha}^{h} \bar{V}_{\beta}^{\bar{h}}|h, \bar{h}\rangle$. Here, $V_{\alpha}^{h}$ and $V_{\beta}^{\bar{h}}$ are polynomials in $L_{-n}$ and $\bar{L}_{-n}$ respectively chosen so that these states give an orthonormal basis of the Verma module: ${ }^{33}$

$$
\left\langle\mathcal{O}_{h, \bar{h}}^{\alpha^{\prime}, \beta^{\prime}}(\infty) \mathcal{O}_{h, \bar{h}}^{\alpha, \beta}(0)\right\rangle=\left\langle h, \bar{h}\left|V_{\alpha^{\prime}}^{h, \dagger} \bar{V}_{\beta^{\prime}}^{\bar{h}, \dagger} V_{\alpha}^{h} \bar{V}_{\beta}^{\bar{h}}\right| h, \bar{h}\right\rangle=\delta_{\alpha \alpha^{\prime}} \delta_{\beta \beta^{\prime}}
$$

We can re-express the same operators in terms of local operators $\mathcal{O}_{h, \bar{h}}^{\alpha_{i}, \beta_{i}}\left(z_{i}, \bar{z}_{i}\right)$ at arbitrary points $z_{1}, z_{2}$ by the use of a global conformal transformation that maps $(\infty, 0)$ to $\left(z_{1}, z_{2}\right)$. We then have

$$
\left\langle\mathcal{O}_{h, \bar{h}}^{\alpha_{1}, \beta_{1}}\left(z_{1}, \bar{z}_{1}\right) \mathcal{O}_{h, \bar{h}}^{\alpha_{2}, \beta_{2}}\left(z_{2}, \bar{z}_{2}\right)\right\rangle=\delta_{\alpha_{1} \alpha_{2}} \delta_{\beta_{1} \beta_{2}} .
$$

Note that the form of each local descendant operator depends explicitly on both points $z_{1}, z_{2}$, and not just implicitly on one point through the local primary. These operators are only orthogonal precisely at the points $z_{1}, z_{2}$ (and form an orthogonal basis of operators in the 'North-South' quantization between these two points).

Next, we require a somewhat more refined version of the doubling trick. We have seen that a correlator

$$
\left\langle\mathcal{O}_{h_{1} \bar{h}_{1}}\left(z_{1}, \bar{z}_{1}\right) \cdots \mathcal{O}_{h_{n} \bar{h}_{n}}\left(z_{n}, \bar{z}_{n}\right)\right\rangle_{\mathrm{UHP}}^{b}
$$

of bulk CFT operators $\mathcal{O}_{h_{k} \bar{h}_{k}}$ with conformal weights $\left(h_{k}, \bar{h}_{k}\right)$ is constrained to have the same functional form as chiral CFT correlators

$$
\left\langle\mathcal{O}_{h_{1}}\left(z_{1}\right) \cdots \mathcal{O}_{h_{n}}\left(z_{n}\right) \mathcal{O}_{\bar{h}_{1}}\left(\bar{z}_{1}\right) \cdots \mathcal{O}_{\bar{h}_{n}}\left(\bar{z}_{n}\right)\right\rangle
$$

Similarly, a correlator of descendants

$$
\left\langle\mathcal{O}_{h_{1} \bar{h}_{1}}^{\alpha_{1}, \beta_{1}}\left(z_{1}, \bar{z}_{1}\right) \cdots \mathcal{O}_{h_{n} \bar{h}_{n}}^{\alpha_{n}, \beta_{n}}\left(z_{n}, \bar{z}_{n}\right)\right\rangle_{\mathrm{UHP}}^{b}
$$

takes the same functional form as

$$
\left\langle\mathcal{O}_{h_{1}}^{\alpha_{1}}\left(z_{1}\right) \cdots \mathcal{O}_{h_{n}}^{\alpha_{n}}\left(z_{n}\right) \mathcal{O}_{\bar{h}_{1}}^{\beta_{1}}\left(\bar{z}_{1}\right) \cdots \mathcal{O}_{\bar{h}_{n}}^{\beta_{n}}\left(\bar{z}_{n}\right)\right\rangle
$$

Then, taking $\mathcal{O}_{h}(z)$ to be a primary operator in some CFT such that ${ }^{34}$

$$
\begin{aligned}
\left\langle\mathcal{O}_{h, h}(z, \bar{z})\right\rangle_{\mathrm{UHP}}^{b} & =\mathcal{A}_{h}^{b}\left\langle\mathcal{O}_{h}(\bar{z}) \mathcal{O}_{h}(z)\right\rangle \\
& =\frac{\mathcal{A}_{h}^{b}}{|z-\bar{z}|^{2 h}}
\end{aligned}
$$

\footnotetext{
${ }^{33}$ Recall that the conjugation operation used to define the dual operator at infinity is an inversion in radial quantization, and so the operator is rescaled by the conformal transformation. We keep the rescaling implicit.

${ }^{34}$ Note that it's not necessary for such a CFT to exist, since we are only making statements about kinematics.
} 
we have that

$$
\begin{aligned}
\left\langle\mathcal{O}_{h, h}^{\alpha, \beta}(z, \bar{z})\right\rangle_{\mathrm{UHP}}^{b} & =\mathcal{A}_{h}^{b}\left\langle\mathcal{O}_{h}^{\alpha}(z) \mathcal{O}_{h}^{\beta}(\bar{z})\right\rangle \\
& =\delta_{\alpha \beta} \mathcal{A}_{h}^{b}
\end{aligned}
$$

where here the descendant indices are labeling the orthogonal basis of states for the pair of points $z, \bar{z}$.

Bulk channel expression for the two-point function. We can now derive a bulkchannel expression for the two-point function (A.5). First we will use the bulk stateoperator map (bulk OPE) to insert a complete set of bulk states (in this 'North-South' quantization between $z_{3}$ and $\bar{z}_{3}$ )

$$
\left\langle\mathcal{O}_{1}\left(z_{1}, \bar{z}_{1}\right) \mathcal{O}_{2}\left(z_{2}, \bar{z}_{2}\right)\right\rangle_{\mathrm{UHP}}^{b}=\sum_{i, \alpha, \beta}\left\langle\mathcal{O}_{1}\left(z_{1}, \bar{z}_{1}\right) \mathcal{O}_{2}\left(z_{2}, \bar{z}_{2}\right) \mathcal{O}_{i}^{\alpha, \beta}\left(\bar{z}_{3}, z_{3}\right)\right\rangle\left\langle\mathcal{O}_{i}^{\alpha, \beta}\left(z_{3}, \bar{z}_{3}\right)\right\rangle_{\mathrm{UHP}}^{b}
$$

Using the form of the boundary one-point function (A.13), we can rewrite this as

$$
\begin{aligned}
\left\langle\mathcal{O}_{1}\left(z_{1}, \bar{z}_{1}\right)\right. & \left.\mathcal{O}_{2}\left(z_{2}, \bar{z}_{2}\right)\right\rangle_{\mathrm{UHP}}^{b} \\
= & \sum_{\left\{i \mid h_{i}=\bar{h}_{i}\right\}, \alpha, \beta} \hat{\mathcal{C}}_{12}^{i} \mathcal{A}_{i}^{b}\left\langle\mathcal{O}_{h_{1}}\left(z_{1}\right) \mathcal{O}_{h_{2}}\left(z_{2}\right) \mathcal{O}_{h_{i}}^{\alpha}\left(\bar{z}_{3}\right)\right\rangle\left\langle\mathcal{O}_{h_{i}}^{\alpha}\left(z_{3}\right) \mathcal{O}_{\bar{h}_{1}}\left(\bar{z}_{1}\right) \mathcal{O}_{\bar{h}_{2}}\left(\bar{z}_{2}\right)\right\rangle,
\end{aligned}
$$

where we have pulled out the dynamical information in the OPE coefficients and expectation values. The three-point functions, as written, are now purely kinematic, i.e. they represent the functional dependence of such a three-point function where the overall coefficient is taken to be one. Each sum over Virasoro descendants now can be seen to give a standard chiral Virasoro conformal block $\mathcal{F}\left(c, h ;\left[h_{1}, h_{2}, \bar{h}_{1}, \bar{h}_{2}\right] \mid z\right)$, so that the two-point function can be expanded in this bulk channel as

$$
\begin{aligned}
\left\langle\mathcal{O}_{1}\left(z_{1}, \bar{z}_{1}\right) \mathcal{O}_{2}\left(z_{2}, \bar{z}_{2}\right)\right\rangle_{\mathrm{UHP}}^{b}= & \left(\frac{z_{21^{*}}}{z_{11^{*}}}\right)^{h_{1}-h_{2}}\left(\frac{z_{11^{*}}}{z_{12^{*}}}\right)^{\bar{h}_{2}-\bar{h}_{1}} \frac{z^{h_{1}+h_{2}}}{\left(z_{12}\right)^{h_{1}+h_{2}\left(z_{2^{*} 1^{*}}\right)^{\bar{h}_{1}+\bar{h}_{2}}}} \\
& \times \sum_{i} \hat{\mathcal{C}}_{12}^{i} \mathcal{A}_{i}^{b} \mathcal{F}\left(c, h_{i} ;\left[h_{1}, h_{2}, \bar{h}_{1}, \bar{h}_{2}\right] \mid z\right),
\end{aligned}
$$

and where we have written the conformal block in terms of the cross-ratio

$$
z=\frac{z_{12} z_{2^{*} 1^{*}}}{z_{12^{*}} z_{21^{*}}} .
$$

Boundary channel expression for the two-point function. We can similarly expand the two-point function in the boundary channel. Here we insert a complete set of states corresponding to the expansion of the bulk operators in terms of the boundary operator expansion. The boundary state-operator mapping gives a complete set of states in terms of boundary operators which appear in representations of the surviving diagonal Virasoro symmetry. We thus insert a complete set of orthonormal states of the form

$$
\left\langle\mathcal{O}_{1}\left(z_{1}, \bar{z}_{1}\right) \mathcal{O}_{2}\left(z_{2}, \bar{z}_{2}\right)\right\rangle_{\mathrm{UHP}}^{b}=\sum_{I, \alpha}\left\langle\mathcal{O}_{1}\left(z_{1}, \bar{z}_{1}\right) \tilde{V}_{\alpha}^{\hat{h}_{I}} \mid \hat{h}_{I}\right\rangle_{\mathrm{UHP}}^{b}\left\langle\hat{h}_{I} \mid \tilde{V}_{\alpha}^{\hat{h}_{I} \dagger} \mathcal{O}_{2}\left(z_{2}, \bar{z}_{2}\right)\right\rangle_{\mathrm{UHP}}^{b} .
$$


Using the doubling trick to account for the representation of bulk operators under the boundary Virasoro operators, we can rewrite this as

$$
\left\langle\mathcal{O}_{1}\left(z_{1}, \bar{z}_{1}\right) \mathcal{O}_{2}\left(z_{2}, \bar{z}_{2}\right)\right\rangle_{\mathrm{UHP}}^{b}=\sum_{I, \alpha} \mathcal{B}_{1 h}^{b} \mathcal{B}_{2 h}^{b}\left\langle\mathcal{O}_{h_{1}}\left(z_{1}\right) \mathcal{O}_{\bar{h}_{1}}\left(z_{1}^{*}\right) V_{\alpha}^{\hat{h}_{I}} \mid \hat{h}_{I}\right\rangle\left\langle\hat{h}_{I} \mid V_{\alpha}^{\hat{h}_{I} \dagger} \mathcal{O}_{h_{2}}\left(z_{2}\right) \mathcal{O}_{\bar{h}_{2}}\left(z_{2}^{*}\right)\right\rangle
$$

where we have pulled out the dynamical information in the coefficients. The remaining three-point functions, as written, are now purely kinematic. Again we recognize that this sum over Virasoro descendants is the standard bulk chiral Virasoro conformal block $\mathcal{F}\left(c, h ;\left[h_{1}, \bar{h}_{1}, h_{2}, \bar{h}_{2}\right] \mid \eta\right)$, giving

$$
\begin{aligned}
& \left\langle\mathcal{O}_{1}\left(z_{1}, \bar{z}_{1}\right) \mathcal{O}_{2}\left(z_{2}, \bar{z}_{2}\right)\right\rangle_{\mathrm{UHP}}^{b}=\left(\frac{z_{1^{*} 2}}{z_{12}}\right)^{h_{1}-\bar{h}_{1}}\left(\frac{z_{12}}{z_{12^{*}}}\right)^{\bar{h}_{2}-h_{2}} \frac{\eta^{h_{1}+\bar{h}_{1}}}{\left(z_{11^{*}}\right)^{h_{1}+\bar{h}_{1}}\left(z_{22^{*}}\right)^{h_{2}+\bar{h}_{2}}} \\
& \times \sum_{I} \mathcal{B}_{1 I}^{b} \mathcal{B}_{2 I}^{b} \mathcal{F}\left(c, h ;\left[h_{1}, \bar{h}_{1}, h_{2}, \bar{h}_{2}\right] \mid \eta\right)
\end{aligned}
$$

where we have used the cross-ratio

$$
\eta=1-z
$$

\section{B Boundary operator expansion for twist operators}

In this section, we relate the boundary operator expansion of the twist operator $\Phi_{n}$ in an $n$-copy BCFT to $n$-point functions of boundary operators in the original BCFT. Our discussion here is directly parallel to the discussion in section 4 of [40] on contributions to the OPE coefficients of CFT twist operators.

Via radial quantization, a twist operator inserted at $z$ into an $n$-copy BCFT can be understood to give rise to some entangled state of this $n$-copy BCFT on an interval. By the state-operator correspondence, the same state can be obtained by the insertion of some operator at the origin. A basis of boundary operators for the $n$-copy BCFT may be written as $\mathcal{O}_{I_{1}} \otimes \cdots \otimes \mathcal{O}_{I_{n}}$, where $\mathcal{O}_{I}$ are a basis of boundary operators in the original BCFT. Thus, we can write that

$$
\Phi_{n}(x+i y)=\sum_{\left\{I_{k}\right\}} \frac{1}{|2 y|^{d_{n}-\sum_{k} \Delta_{I_{k}}}} B_{I_{1} \cdots I_{n}}^{\Phi_{n}} \mathcal{O}_{I_{1}} \otimes \cdots \otimes \mathcal{O}_{I_{n}}(x)
$$

When the operators $\mathcal{O}_{I_{1}}$ are primary, the coefficient $B_{I_{1} \cdots I_{n}}^{\Phi_{n}}$ can be defined according to (2.13) via the bulk-boundary two-point function as

$$
\begin{aligned}
B_{I_{1} \cdots I_{n}}^{\Phi_{n}} & =2^{d_{n}-\sum_{k} \Delta_{I_{k}}}\left\langle\Phi_{n}(z=i) \mathcal{O}_{I_{1}} \otimes \cdots \otimes \mathcal{O}_{I_{n}}(0)\right\rangle \\
& =2^{d_{n}-\sum_{k} \Delta_{I_{k}}}\left\langle\Phi_{n}(i)\right\rangle \frac{\left\langle\Phi_{n}(i) \mathcal{O}_{I_{1}} \otimes \cdots \otimes \mathcal{O}_{I_{n}}(0)\right\rangle}{\left\langle\Phi_{n}(i)\right\rangle} \\
& =2^{-\sum_{k} \Delta_{I_{k}} \epsilon^{d_{n}}} g_{b}^{1-n} \frac{\left\langle\Phi_{n}(i) \mathcal{O}_{I_{1}} \otimes \cdots \otimes \mathcal{O}_{I_{n}}(0)\right\rangle}{\left\langle\Phi_{n}(i)\right\rangle} .
\end{aligned}
$$

To compute the ratio of correlators in the last line, consider the conformal transformation

$$
w(z)=i\left(\frac{(z+i)^{n}+(z-i)^{n}}{(z+i)^{n}-(z-i)^{n}}\right) .
$$


This takes the UHP to the $n$-sheeted UHP associated with the insertion of our twist operator. The points

$$
x_{k} \equiv \cot \left(\pi \frac{2 k-1}{2 n}\right) \quad k=1, \ldots, n
$$

map to the origin on the various sheets. By this conformal transformation, we have that

$$
\frac{\left\langle\Phi_{n}(i) \mathcal{O}_{I_{1}} \otimes \cdots \otimes \mathcal{O}_{I_{n}}(0)\right\rangle}{\left\langle\Phi_{n}(i)\right\rangle}=\prod_{k}\left(\frac{\mathrm{d} w}{\mathrm{~d} z}\left(x_{k}\right)\right)^{-\Delta_{I_{k}}}\left\langle\prod_{k} \mathcal{O}_{I_{k}}\left(x_{k}\right)\right\rangle
$$

For the points $x_{k}$ where $w(z)=0$, we have that

$$
\frac{\mathrm{d} w}{\mathrm{~d} z}\left(x_{k}\right)=\frac{n}{x_{k}^{2}+1}=n \sin ^{2}\left(\pi \frac{2 k-1}{2 n}\right)
$$

Combining everything, we have that

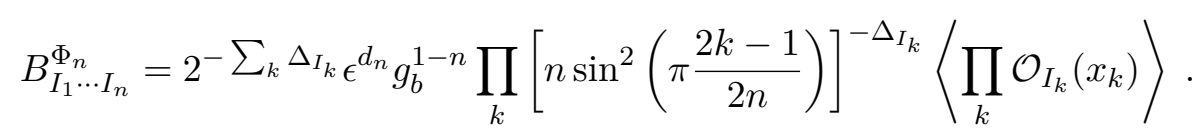

It is useful to note that the explicit dependence on $c$ appears as a universal prefactor,

$$
B_{I_{1} \cdots I_{n}}^{\Phi_{n}}=\epsilon^{d_{n}} g_{b}^{1-n} \bar{B}_{I_{1} \cdots I_{n}}^{\Phi_{n}}
$$

For $n=2$, we see that the correlator vanishes unless $I_{1}=I_{2}$, and we have that

$$
\bar{B}_{I I}^{\Phi_{2}}=\frac{1}{16^{\Delta_{I}}} .
$$

For $n=3$, we have that

$$
\bar{B}_{I J K}^{\Phi_{3}}=\frac{C_{I J K}}{3^{\frac{3}{2}\left(\Delta_{I}+\Delta_{J}+\Delta_{K}\right)}},
$$

where we have used the standard result for a CFT three-point function.

\section{Monodromy method}

Here we continue the discussion of accessory parameters from (5.9) in the main text to give a more complete description of the calculation of the semiclassical blocks.

There are only $2 k-3$ independent accessory parameters, since global $\operatorname{SL}(2, \mathbb{R})$ invariance imposes three (real) constraints. Explicitly, these constraints are

$$
\sum_{i} \Re\left(c_{i}\right)=\sum_{i} \Re\left(c_{i} z_{i}-\frac{6 h_{n}}{c}\right)=\sum_{i} \Re\left(c_{i} z_{i}^{2}-\frac{12 h_{n} z_{i}}{c}\right)=0,
$$

the real part of the usual $\mathrm{SL}(2, \mathbb{C})$ constraints.

If we know the accessory parameters, we can integrate to find the block $f_{0}^{\mathcal{E}}$. To determine these parameters, we transport a pair of solutions $\vec{\Theta}(z)=\left[\Theta^{+}(z), \Theta^{-}(z)\right]^{T}$ around a point $z_{c}$ where an OPE or BOE is to be performed. The null decoupling equation (5.4) 
applied to the three-point function implies that the $2 \times 2$ monodromy matrix $M$ performing the transport, $\vec{\Theta}(z) \mapsto M \vec{\Theta}(z)$, gives $^{35}$

$$
\operatorname{tr} M=-2 \cos \left(\pi \Lambda_{c}\right), \quad \Lambda_{c}=\sqrt{1-\frac{24 h_{n}}{c}} .
$$

The number of independent monodromies to tune equals the number of internal primaries, $2 k-3,{ }^{36}$ so we have the right number of monodromy constraints to fix our accessory parameters $c_{i}$.

In general, we cannot analytically solve for the accessory parameters. Luckily, however, it is possible to find them explicitly for twist operators in the $n \rightarrow 1$ limit. As above, we define $\alpha=(n-1) / 12$. Entanglement entropy is obtained from Rényi entropies in the limit $\alpha \rightarrow 0$, and since $h_{n}=c(n+1) \alpha / 2 n=c \alpha \rightarrow 0$ in this limit, the function (5.8) vanishes away from the singular points $z_{i}, \bar{z}_{i}$. As a result, the equation (5.4) decouples into a sum of independent monodromy equations, depending on which cycles the channel $\mathcal{E}$ trivializes.

To illustrate, suppose $\mathcal{E}$ involves a pairing between twists $\Phi_{n}\left(z_{i}\right)$ and $\bar{\Phi}_{n}\left(z_{j}\right)$. We must choose the accessory parameters to make the monodromy around $z_{i}, z_{j}$ trivial. Since this decouples from the other problems as $\alpha \rightarrow 0$, we can simply focus on the contribution

$$
\begin{aligned}
T_{i j}(z) & =\frac{6 h_{n}}{c}\left[\frac{1}{\left(z-z_{i}\right)^{2}}+\frac{1}{\left(z-z_{j}\right)^{2}}\right]-\frac{c_{i}}{z-z_{i}}-\frac{c_{j}}{z-z_{j}}+\text { c.c. } \\
& =6 \alpha\left[\frac{1}{\left(z-z_{i}\right)^{2}}+\frac{1}{\left(z-z_{j}\right)^{2}}-\frac{2}{z_{j}\left(z-z_{i}\right)}\right]-\frac{c_{i}}{z-z_{i}}+\frac{c_{i} z_{i}}{z_{j}\left(z-z_{j}\right)}+\text { c.c. }
\end{aligned}
$$

where "c.c" stands for complex conjugate terms, and in (C.2), we used the constraint $\Re\left(c_{i} z_{i}+c_{j} z_{j}\right)=6 \alpha$. To obtain a trivial monodromy around $z_{i}, z_{j}$ (and the image cycle enclosing $\left.\bar{z}_{i}, \bar{z}_{j}\right)$, it is sufficient for $T_{i j}(z)$ to be regular at infinity. This is equivalent to the sum of residues at simple poles vanishing, and hence

$$
c_{i}+\bar{c}_{i}=\frac{12 \alpha}{\left|z_{i j}\right|^{2}}+O\left(\alpha^{2}\right)
$$

where $O\left(\alpha^{2}\right)$ corrections arise because the equations only strictly decouple for $\alpha=0$. The calculation is analogous for a twist paired with its image, but the contribution $T_{m m^{*}}(z)$ involves only two insertions at $z_{m}$ and $\bar{z}_{m}$.

If we integrate the accessory parameters defined in (C.3) (and the image-paired counterparts), we find

$$
f_{0}^{\mathcal{E}}=\sum_{(i j)} 12 \alpha \log \left|z_{i j}\right|^{2}+\sum_{\left(m m^{*}\right)} 12 \alpha \log z_{m m^{*}}+O\left(\alpha^{2}\right)
$$

as required.

\footnotetext{
${ }^{35}$ To see this, we suppose the leading term in $\Theta(z) \sim\left(z-z_{c}\right)^{\kappa}$. Plugging this into (5.4), we find that $\kappa(\kappa-1)=-6 h_{n} / c$, with two solutions $\kappa_{ \pm}$. These pick up factors $e^{2 \pi i \kappa_{ \pm}}$after traversing a loop $z=z_{c}+\epsilon e^{i \theta}$, leading to (C.1). See [10] for details.

${ }^{36}$ An exchange channel $\mathcal{E}$ is a cubic tree with $2 k$ leaves and $2 k-2$ internal nodes in the doubled picture. The total number of edges is one less than the number of nodes, $E=4 k-3$, and hence the number of internal edges is $E-2 k=2 k-3$.
} 
Open Access. This article is distributed under the terms of the Creative Commons Attribution License (CC-BY 4.0), which permits any use, distribution and reproduction in any medium, provided the original author(s) and source are credited.

\section{References}

[1] S. Ryu and T. Takayanagi, Aspects of holographic entanglement entropy, JHEP 08 (2006) 045 [hep-th/0605073] [INSPIRE].

[2] A. Karch and L. Randall, Open and closed string interpretation of SUSY CFT's on branes with boundaries, JHEP 06 (2001) 063 [hep-th/0105132] [INSPIRE].

[3] T. Takayanagi, Holographic dual of BCFT, Phys. Rev. Lett. 107 (2011) 101602 [arXiv:1105.5165] [INSPIRE].

[4] T. Anous, T. Hartman, A. Rovai and J. Sonner, Black hole collapse in the $1 /$ c expansion, JHEP 07 (2016) 123 [arXiv: 1603.04856] [INSPIRE].

[5] S. Cooper, M. Rozali, B. Swingle, M. Van Raamsdonk, C. Waddell and D. Wakeham, Black hole microstate cosmology, JHEP 07 (2019) 065 [arXiv:1810.10601] [INSPIRE].

[6] M. Rozali, J. Sully, M. Van Raamsdonk, C. Waddell and D. Wakeham, Information radiation in BCFT models of black holes, JHEP 05 (2020) 004 [arXiv: 1910.12836] [INSPIRE].

[7] G. Penington, Entanglement wedge reconstruction and the information paradox, JHEP 09 (2020) 002 [arXiv: 1905. 08255] [InSPIRE].

[8] A. Almheiri, N. Engelhardt, D. Marolf and H. Maxfield, The entropy of bulk quantum fields and the entanglement wedge of an evaporating black hole, JHEP 12 (2019) 063 [arXiv: 1905.08762] [INSPIRE].

[9] A. Almheiri, R. Mahajan, J. Maldacena and Y. Zhao, The Page curve of Hawking radiation from semiclassical geometry, JHEP 03 (2020) 149 [arXiv: 1908.10996] [INSPIRE].

[10] T. Hartman, Entanglement entropy at large central charge, arXiv:1303.6955 [INSPIRE].

[11] T. Faulkner, The entanglement Renyi entropies of disjoint intervals in AdS/CFT, arXiv: 1303.7221 [INSPIRE].

[12] A. Almheiri, T. Hartman, J. Maldacena, E. Shaghoulian and A. Tajdini, Replica wormholes and the entropy of Hawking radiation, JHEP 05 (2020) 013 [arXiv:1911.12333] [INSPIRE].

[13] G. Penington, S.H. Shenker, D. Stanford and Z. Yang, Replica wormholes and the black hole interior, arXiv:1911.11977 [INSPIRE].

[14] D.M. McAvity and H. Osborn, Conformal field theories near a boundary in general dimensions, Nucl. Phys. B 455 (1995) 522 [cond-mat/9505127] [INSPIRE].

[15] P. Di Francesco, P. Mathieu and D. Senechal, Conformal field theory, Graduate Texts in Contemporary Physics, Springer, Germany (1997) [InSPIRE].

[16] J.L. Cardy, Boundary conformal field theory, hep-th/0411189 [INSPIRE].

[17] P. Calabrese and J. Cardy, Entanglement entropy and conformal field theory, J. Phys. A 42 (2009) 504005 [arXiv: 0905.4013] [InSPIRE].

[18] P. Liendo, L. Rastelli and B.C. van Rees, The bootstrap program for boundary $C F T_{d}, J H E P$ 07 (2013) 113 [arXiv: 1210.4258] [INSPIRE]. 
[19] P. Calabrese and J. Cardy, Quantum quenches in $1+1$ dimensional conformal field theories, J. Stat. Mech. 1606 (2016) 064003 [arXiv:1603.02889] [INSPIRE].

[20] I. Affleck and A.W.W. Ludwig, Universal noninteger 'ground state degeneracy' in critical quantum systems, Phys. Rev. Lett. 67 (1991) 161 [INSPIRE].

[21] J.L. Cardy, Boundary conditions, fusion rules and the Verlinde formula, Nucl. Phys. B 324 (1989) 581 [INSPIRE].

[22] A. Recknagel and V. Schomerus, Boundary conformal field theory and the worldsheet approach to D-branes, Cambridge Monographs on Mathematical Physics, Cambridge University Press, Cambridge U.K. (2013) [InSPIRE].

[23] M. Fujita, T. Takayanagi and E. Tonni, Aspects of AdS/BCFT, JHEP 11 (2011) 043 [arXiv:1108.5152] [INSPIRE].

[24] A. Faraji Astaneh and S.N. Solodukhin, Holographic calculation of boundary terms in conformal anomaly, Phys. Lett. B 769 (2017) 25 [arXiv:1702.00566] [InSPIRE].

[25] M. Chiodaroli, E. D'Hoker and M. Gutperle, Simple holographic duals to boundary CFTs, JHEP 02 (2012) 005 [arXiv:1111.6912] [INSPIRE].

[26] M. Chiodaroli, E. D'Hoker and M. Gutperle, Holographic duals of Boundary CFTs, JHEP 07 (2012) 177 [arXiv:1205.5303] [INSPIRE].

[27] E. D'Hoker, J. Estes and M. Gutperle, Exact half-BPS type IIB interface solutions. I. Local solution and supersymmetric Janus, JHEP 06 (2007) 021 [arXiv:0705.0022] [INSPIRE].

[28] E. D'Hoker, J. Estes and M. Gutperle, Exact half-BPS type IIB interface solutions. II. Flux solutions and multi-Janus, JHEP 06 (2007) 022 [arXiv:0705.0024] [INSPIRE].

[29] O. Aharony, L. Berdichevsky, M. Berkooz and I. Shamir, Near-horizon solutions for D3-branes ending on 5-branes, Phys. Rev. D 84 (2011) 126003 [arXiv:1106.1870] [INSPIRE].

[30] B. Assel, C. Bachas, J. Estes and J. Gomis, Holographic duals of $D=3 N=4$ superconformal field theories, JHEP 08 (2011) 087 [arXiv:1106.4253] [INSPIRE].

[31] D. Seminara, J. Sisti and E. Tonni, Corner contributions to holographic entanglement entropy in $A d S_{4} / B C F T_{3}, J H E P 11$ (2017) 076 [arXiv:1708.05080] [INSPIRE].

[32] A. Almheiri, A. Mousatov and M. Shyani, Escaping the interiors of pure boundary-state black holes, arXiv: 1803.04434 [INSPIRE].

[33] J.L. Cardy, O.A. Castro-Alvaredo and B. Doyon, Form factors of branch-point twist fields in quantum integrable models and entanglement entropy, J. Statist. Phys. 130 (2008) 129 [arXiv:0706.3384] [INSPIRE].

[34] J. Cardy and E. Tonni, Entanglement hamiltonians in two-dimensional conformal field theory, J. Stat. Mech. 1612 (2016) 123103 [arXiv:1608.01283] [InSPIRE].

[35] A.A. Belavin, A.M. Polyakov and A.B. Zamolodchikov, Infinite conformal symmetry in two-dimensional quantum field theory, Nucl. Phys. B 241 (1984) 333 [InSPIRE].

[36] H. Dorn and H.J. Otto, Two and three point functions in Liouville theory, Nucl. Phys. B 429 (1994) 375 [hep-th/9403141] [INSPIRE].

[37] A.B. Zamolodchikov and A.B. Zamolodchikov, Structure constants and conformal bootstrap in Liouville field theory, Nucl. Phys. B 477 (1996) 577 [hep-th/9506136] [InSPIRE]. 
[38] D. Harlow, J. Maltz and E. Witten, Analytic continuation of Liouville theory, JHEP 12 (2011) 071 [arXiv: 1108.4417] [INSPIRE].

[39] A.B. Zamolodchikov, Conformal symmetry in two-dimensional space: recursion representation of conformal block, Theor. Math. Phys. 73 (1987) 1088.

[40] E. Perlmutter, Comments on Renyi entropy in $A d S_{3} / C F T_{2}$, JHEP 05 (2014) 052 [arXiv: 1312.5740] [INSPIRE].

[41] D. Pappadopulo, S. Rychkov, J. Espin and R. Rattazzi, OPE convergence in conformal field theory, Phys. Rev. D 86 (2012) 105043 [arXiv:1208.6449] [InSPIRE].

[42] B. Chen and J.-J. Zhang, On short interval expansion of Rényi entropy, JHEP 11 (2013) 164 [arXiv: 1309.5453] [INSPIRE].

[43] P. Calabrese, J. Cardy and E. Tonni, Entanglement entropy of two disjoint intervals in conformal field theory II, J. Stat. Mech. 1101 (2011) P01021 [arXiv:1011.5482] [INSPIRE].

[44] G.H. Hardy and S. Ramanujan, Asymptotic formulace in combinatory analysis, Proc. London Math. Soc. s2-17 (1918) 75.

[45] E. Shaghoulian, Emergent gravity from Eguchi-Kawai reduction, JHEP 03 (2017) 011 [arXiv: 1611.04189] [INSPIRE].

[46] T. Hartman, C.A. Keller and B. Stoica, Universal spectrum of $2 d$ conformal field theory in the large c limit, JHEP 09 (2014) 118 [arXiv:1405.5137] [INSPIRE]. 